\title{
X-band microwave characterisation and analysis of carbon fibre-reinforced polymer composites
}

DOI:

10.1016/j.compstruct.2018.09.099

\section{Document Version}

Accepted author manuscript

Link to publication record in Manchester Research Explorer

\section{Citation for published version (APA):}

Li, Z., Haigh, A., Soutis, C., \& Gibson, A. (2019). X-band microwave characterisation and analysis of carbon fibrereinforced polymer composites. Composite Structures, 208, 224-232.

https://doi.org/10.1016/j.compstruct.2018.09.099

\section{Published in:}

Composite Structures

\section{Citing this paper}

Please note that where the full-text provided on Manchester Research Explorer is the Author Accepted Manuscript or Proof version this may differ from the final Published version. If citing, it is advised that you check and use the publisher's definitive version.

\section{General rights}

Copyright and moral rights for the publications made accessible in the Research Explorer are retained by the authors and/or other copyright owners and it is a condition of accessing publications that users recognise and abide by the legal requirements associated with these rights.

\section{Takedown policy}

If you believe that this document breaches copyright please refer to the University of Manchester's Takedown Procedures [http://man.ac.uk/04Y6Bo] or contact uml.scholarlycommunications@manchester.ac.uk providing relevant details, so we can investigate your claim.

\section{OPEN ACCESS}




\section{Manuscript Details}

\section{Manuscript number}

Title
COST_2018_2383

$\mathrm{X}$-band microwave characterisation and analysis of carbon fibre-reinforced polymer composites

Full Length Article

\section{Article type}

Abstract

In this paper, a detailed investigation of the electromagnetic (EM) properties of carbon fibre-reinforced polymer composites (CFRP) is presented. The electric permittivity, electrical conductivity, signal penetration and microwave absorption are discussed. Unidirectional composite laminate samples were characterised over $\mathrm{X}$ band $(8-12 \mathrm{GHz})$ using the microwave transmission line technique. It is shown that the real part of the permittivity of the composites is not significantly anisotropic whereas the imaginary part is highly anisotropic. More microwave energy is reflected in the parallel case, while more energy is absorbed in the orthogonal case. These experimental results are studied at three geometric levels: macro-meso scale (laminate/lamina level) relating to lay-up and fibre direction dependence, microscale (fibre level) relating to the real part of permittivity and nanoscale level relating to imaginary part of permittivity. The findings can contribute to improved design of carbon fibre composites for electromagnetic applications, like shielding, curing and non-destructive inspection.

\section{Keywords}

Corresponding Author

Corresponding Author's Institution

Order of Authors

\section{Suggested reviewers}

carbon fibre composites; electromagnetic properties; microwave characterisation; multiscale analysis; microwave absorption.

\section{Constantinos Soutis}

The University of Manchester

Zhen Li, Arthur Haigh, Constantinos Soutis, Andrew Gibson

Adrian Porch, Bartlomiej Salski, Christian Boller 


\section{Submission Files Included in this PDF}

\section{File Name [File Type]}

Cover_letter_em_cost.doc [Cover Letter]

EM_composites_manuscript.docx [Manuscript File]

fig1.tif [Figure]

fig2.tif [Figure]

fig3a.tif [Figure]

fig3b.tif [Figure]

fig4a.tif [Figure]

fig4b.tif [Figure]

fig5a.tif [Figure]

fig5b.tif [Figure]

fig5c.tif [Figure]

fig5d.tif [Figure]

fig6.tif [Figure]

fig7a.tif [Figure]

fig7b.tif [Figure]

fig8.tif [Figure]

fig9.tif [Figure]

fig10a.tif [Figure]

fig10b.tif [Figure]

fig11.tif [Figure]

fig12.tif [Figure]

fig13.tif [Figure]

fig14.tif [Figure]

fig15.tif [Figure]

To view all the submission files, including those not included in the PDF, click on the manuscript title on your EVISE Homepage, then click 'Download zip file'. 


\section{Cover letter for submission of a paper to Composite Structures}

Dear Prof. A.J.M.Ferreira,

We wish to submit a new manuscript entitled "X-band microwave characterisation and analysis of carbon fibre-reinforced polymer composites" for your consideration.

We confirm that this work is original and has not been published elsewhere nor is it currently under consideration for publication elsewhere.

In this paper, we report a detailed investigation of the electromagnetic (EM) properties of carbon fibre-reinforced polymer composites (CFRP). The electric permittivity, electrical conductivity, signal penetration and microwave absorption are discussed. Unidirectional composite laminate samples were characterised over $\mathrm{X}$ band (8-12 GHz) using the microwave transmission line technique. It is shown that the real part of the permittivity of the composites is not significantly anisotropic whereas the imaginary part is highly anisotropic. More microwave energy is reflected in the parallel case, while more energy is absorbed in the orthogonal case. These experimental results are studied at three geometric levels: macro-meso scale (laminate/lamina level) relating to lay-up and fibre direction dependence, microscale (fibre level) relating to the real part of permittivity and nanoscale level relating to imaginary part of permittivity. The findings can contribute to improved design of carbon fibre composites for electromagnetic applications, like shielding, curing and non-destructive inspection.

The paper should be of interest to readers in the area of study of dielectric properties of carbon fibre composite structures.

Thank you for your consideration of this manuscript.

Sincerely,

Constantinos Soutis

Aerospace Research Institute, The University of Manchester

James Lighthill Building, Sackville Street, Manchester M13 9PL, UK

Email: constantinos.soutis@manchester.ac.uk 
$\mathrm{X}$-band microwave characterisation and analysis of carbon fibre-reinforced polymer composites Zhen Li a,b, Arthur Haigh c , Constantinos Soutis b,*, Andrew Gibson d

${ }^{a}$ College of Automation Engineering, Nanjing University of Aeronautics and Astronautics, Nanjing 211106, China

${ }^{\mathrm{b}}$ Aerospace Research Institute, The University of Manchester, Manchester, M13 9PL, UK ${ }^{\mathrm{c}}$ School of Electrical and Electronic Engineering, The University of Manchester, Manchester, M13 9PL, UK

${ }^{\mathrm{d}}$ Faculty of Science and Engineering, Manchester Metropolitan University, Manchester, M1 5GD, UK

*Corresponding author: constantinos.soutis@manchester.ac.uk

\begin{abstract}
In this paper, a detailed investigation of the electromagnetic (EM) properties of carbon fibrereinforced polymer composites (CFRP) is presented. The electric permittivity, electrical conductivity, signal penetration and microwave absorption are discussed. Unidirectional composite laminate samples were characterised over $\mathrm{X}$ band $(8-12 \mathrm{GHz})$ using the microwave transmission line technique. It is shown that the real part of the permittivity of the composites is not significantly anisotropic whereas the imaginary part is highly anisotropic. More microwave energy is reflected in the parallel case, while more energy is absorbed in the orthogonal case. These experimental results are studied at three geometric levels: macro-meso scale (laminate/lamina level) relating to lay-up and fibre direction dependence, microscale (fibre level) relating to the real part of permittivity and nanoscale level relating to imaginary part of permittivity. The findings can contribute to improved design of carbon fibre composites for electromagnetic applications, like shielding, curing and nondestructive inspection.
\end{abstract}

\title{
Keywords
}

carbon fibre composites, electromagnetic properties, microwave characterisation, multiscale analysis, microwave absorption 


\section{Introduction}

Carbon fibre-reinforced polymer (CFRP) composites have been increasingly used in aircraft [1]. Compared with the traditional aluminium construction, the fibre-resin composite provides good fatigue and corrosion resistance and a high strength-to-weight ratio that enables fuel savings. Carbon fibres are usually produced from natural cellulose, synthetic polyacrylonitrile (PAN) and pitch, by carbonisation and/or graphitisation at high temperatures to eliminate other chemical elements and generate polycrystalline graphitic structures [2]. In general, fibres with a diameter of 5 to $10 \mu \mathrm{m}$ are the primary load-carrying members with high strength and Young's modulus, while the surrounding matrix acts as a load transfer medium and keeps the fibres in the desired location and orientation [3]. It is noted that most of the composites research is focused on the mechanical properties, which have been thoroughly studied and well documented. In comparison, the work on radar interference, radar cross section (RCS), lightning discharge and electromagnetic shielding were conducted by only measuring the electromagnetic (EM) responses (e.g., reflectance and absorption) of the composite structure [4-6]. The EM properties (or dielectric properties) of the materials were not fully investigated, which impedes their full use for radio frequency (RF) applications.

The electric permittivity $\varepsilon$ and magnetic permeability $\mu$ are the two main parameters of EM properties, which describe the interaction between the material and the EM field. CFRP is nonmagnetic, and its permeability is equal to that of free space (i.e., $\mu_{0}=4 \pi \times 10^{-7} \mathrm{H} \cdot \mathrm{m}^{-1}$ ). Hence permittivity is the parameter of interest in the present work. The electrical conductivity can be deduced from the imaginary part of the permittivity. From a measurement point of view, any material can be characterised by the complex permittivity [7]. Different from glass fibre composites, there is no empirical model available for permittivity prediction of continuous carbon fibre composites. Hence, the investigation on permittivity shall be carried out preferably using experimental techniques. The waveguide technique/transmission line method is one of the commonly used permittivity measurement methods, where the samples under test should fit inside the waveguide. 
Much research has been done on the permittivity measurement of carbon-based nanocomposites (i.e., epoxy resin infused with nanoparticles like graphene, graphite, carbon black, carbon nanofibers (CNF) and carbon nanotubes (CNT)) [8-12]. These nanocomposites can provide better microwave absorption, while this type of composites is not used for aircraft components, thus not in the scope of this paper. At present, very few papers can be found on the measurement of the dielectric properties of continuous carbon fibre composites. Some tests and electromagnetic modelling of the composites presented in the literature were flawed. As seen in $[13,14]$, the dimensions of the composite panel were larger than those of the waveguide, and the panel was clamped between two waveguide sections. Within this arrangement the flow of the surface current on the waveguide walls was disrupted, and the electromagnetic waves were not confined within the waveguide as required. In that case the measurement accuracy could be compromised. In [15], the permittivity of an IM7/977-3 CFRP laminate with two fibre directions with respect to the incident electric field was measured. But the mechanism behind the differences in the permittivity obtained due to the fibre direction was not explained. In [16,17], the conductivity obtained from the direct current (DC) measurement (twoprobe and four-probe methods) was directly used for design of dipole antenna, while in fact the conductivity is frequency-dependent according to the Drude theory [18]. In Reference [19], the CFRP composite laminate was simulated as a number of conductive and dielectric layers alternately stacked together, which does not accurately represent the nature of the real structure. In order to achieve better understanding of the electromagnetic performance of continuous carbon fibre composites, here their electromagnetic properties are investigated in detail. A CFRP unidirectional (UD) laminate was fabricated and electrically characterised over $\mathrm{X}$ band (8-12 GHz) using the microwave transmission line technique. First, the concept of the electric permittivity for description of electromagnetic properties is introduced. Then fabrication process of the composite panel, the experimental setup and permittivity calculation procedure are presented. The permittivity, electrical conductivity, signal penetration and energy absorption of the neat resin and the CFRP 
samples with fibres in orthogonal orientations (i.e., parallel and perpendicular to the incident electric field vector) are examined. Three geometric scales are adopted in the permittivity analysis to thoroughly study the differences in the electromagnetic responses caused by the fibre direction.

\section{Electromagnetic characteristics of materials}

The permittivity of a material can be written as

$$
\varepsilon=\varepsilon_{0} \varepsilon_{\mathrm{r}}=\varepsilon_{0}\left(\varepsilon_{\mathrm{r}}^{\prime}-j \varepsilon_{\mathrm{r}}^{\prime \prime}\right)
$$

where $\varepsilon_{0}$ is the permittivity of free space (i.e., $8.8542 \times 10^{-12} \mathrm{~F} \cdot \mathrm{m}^{-1}$ ), and $\varepsilon_{r}$ is the relative permittivity. The real part $\varepsilon_{\mathrm{r}}^{\prime}$, or dielectric constant, is related to the ability of a material to store the electric field energy, while dielectric loss factor $\varepsilon_{\mathrm{r}}^{\prime \prime}$ accounts for the dissipation of the energy within the material in the form of heat. $\varepsilon_{\mathrm{r}}^{\prime \prime}$ is positive due to energy conservation.

Within the material, bound charges and free charges exist, and motion of the bound charge results in polarisation. For dielectrics, several polarisation mechanisms are involved: ionic and dipolar relaxation, and atomic and electronic resonances at higher frequencies. Over the microwave range, the atomic and electronic polarisations are relatively weak [20], and the ionic conduction and dipole rotation are the dominant loss mechanisms:

$$
\begin{gathered}
\varepsilon_{\mathrm{r}}^{\prime \prime}=\varepsilon_{\mathrm{d}}^{\prime \prime}+\varepsilon_{\sigma}^{\prime \prime}+\varepsilon_{a}^{\prime \prime}+\varepsilon_{e}^{\prime \prime} \approx \varepsilon_{\mathrm{d}}^{\prime \prime}+\varepsilon_{\sigma}^{\prime \prime} \text { (microwave range) } \\
\varepsilon_{\sigma}^{\prime \prime}=\frac{\sigma}{\omega \varepsilon_{0}}
\end{gathered}
$$

where subscripts "d", " $\sigma "$, "a" and "e" denote the contributions due to the dipole rotation, ionic conduction, atomic and electronic resonances, respectively; " $\sigma$ " is the ionic conductivity (in $\mathrm{S} / \mathrm{m}$ ) of a material, $\omega=2 \pi f$ is the angular frequency in $\mathrm{rad} / \mathrm{s}$ and $f$ is the frequency in Hz. Here $\sigma$ is the frequency-dependent alternating current (AC) conductivity, which is different from the DC conductivity $[18,21]$. 
According to the Maxwell's curl equation,

$$
\nabla \times \overline{\mathrm{H}}=j \omega \varepsilon_{0}\left(\varepsilon_{\mathrm{r}}^{\prime}-j \varepsilon_{\mathrm{d}}^{\prime \prime}-j \frac{\sigma}{\omega \varepsilon_{0}}\right) \overline{\mathrm{E}}
$$

where $\overline{\mathrm{H}}$ is the magnetic field in the medium. It is observed that the loss due to the dielectric damping $\left(\varepsilon_{\mathrm{d}}^{\prime \prime}\right)$ is indistinguishable from the conductivity loss $(\sigma)$. The term $\omega \varepsilon_{0} \varepsilon_{\mathrm{d}}^{\prime \prime}+\sigma$ can be considered as the total effective conductivity $\sigma_{\text {eff }}[22]$, and the dielectric loss factor becomes:

$$
\varepsilon_{\mathrm{r}}^{\prime \prime}=\varepsilon_{\mathrm{d}}^{\prime \prime}+\frac{\sigma}{\omega \varepsilon_{0}}=\frac{\sigma_{\text {eff }}}{\omega \varepsilon_{0}}
$$

As the microwaves travel in the medium, the electric field energy decreases as a function of the distance from the surface. A practical parameter used for evaluation of the signal propagation is the penetration depth $d_{p}$, which is defined as the depth where the magnitude is reduced to $1 / e$ (about 37 $\%)$. For a plane wave incident on a half space of a medium, $d_{\mathrm{p}}$ can be given by [23]:

$$
d_{p}=\frac{c}{\sqrt{2} \pi f\left\{\varepsilon_{r}^{\prime}\left[\sqrt{1+\left(\frac{\varepsilon_{r}^{\prime \prime}}{\varepsilon_{r}^{\prime}}\right)^{2}}-1\right]\right\}^{1 / 2}}
$$

where $c$ is the speed of light in free space.

Most metals can be categorised as good conductors. In terms of $\varepsilon$, this condition is equivalent to $\varepsilon_{\mathrm{r}}^{\prime \prime} \gg \varepsilon_{r}^{\prime}$. In this case, $d_{p}$ can be approximated by [22]:

$$
d_{p} \cong \sqrt{\frac{2}{\omega \mu \sigma}}=\frac{1}{\sqrt{\pi f \mu \sigma}}
$$

It is seen that the penetration depth approaches zero when $\varepsilon_{\mathrm{r}}^{\prime \prime}$ becomes very large, thus these metals are good reflectors to microwaves. Common dielectric materials have a very low $\varepsilon_{\mathrm{r}}^{\prime \prime}$, which contributes to a high penetration depth and little energy absorption. This kind of materials can be viewed as transparent to microwaves. However, a combination of metals and low-loss dielectrics, 
like polymer infused with metallic, carbon nanoparticles or long carbon fibres, can result in a material that exhibits medium reflectance and medium transparency and behaves like a lossy dielectric. The material of interest in the present work falls into the last category. As shown in Fig. 1, the microwave energy is most absorbed in the material with a $\varepsilon_{\mathrm{r}}^{\prime \prime}$ value in the middle range [24].

\section{Microwave characterisation of composites}

\subsection{Sample fabrication}

First, as shown in Fig. 2, ten layers of T300 non-woven carbon fibre unidirectional cloth were stacked on a cleaned aluminium plate (mould). Then a sheet of release peel ply was draped over the top of the preform. In order to make the resin flow in a uniform manner, a spiral tube was placed onto the peel ply on each side of the mould. One end of each tube was connected to a plastic tube as resin inlet/outlet. A sheet of infusion mesh was put over the peel ply to improve the resin distribution across the preform. Vacuum bagging film was used to cover the mould with tacky tape. The epoxy resin was made of Aradite LY564 and hardener Aradur HY 2954 with the recommended mix ratio of 100:35 by weight. The composite fabric was infused with epoxy resin by utilising the vacuumassisted resin transfer moulding (VARTM) technique [25]. The whole assembly was cured in an oven at $80^{\circ} \mathrm{C}$ for eight hours, following the manufacturer's guidelines. Subsequently, the composite laminate was demoulded and cut into test pieces.

\subsection{Experimental setup}

The microwave characterisation of the samples was performed as schematically illustrated in Fig. 3. The HP 8510C Vector Network Analyser (VNA) was calibrated before the test using the thru-reflectline (TRL) standard. Calibration accuracy was checked using a flush short (a $6.24 \mathrm{~mm}$ thick copper sheet). Over the frequency range the attenuation was less than $0.1 \mathrm{~dB}$, and the phase shift was $180^{\circ} \pm$ $0.5^{\circ}$. After the calibration, an S-parameter measurement was carried out at the indicated reference 
planes. A personal computer (PC) was connected to the analyser by an IEEE-488 cable. A MATLAB ${ }^{\circledR}$ programme was developed for data acquisition and permittivity calculation.

Two samples with the inner dimensions of the rectangular waveguide were prepared. As shown in Fig. 4, in one sample the fibres were parallel to the electric field vector ("parallel case"), while in the other sample the fibres were orthogonal to the vector ("orthogonal case"). A neat resin sample cured under the same conditions as the composite was measured for comparison purposes.

\subsection{Electric permittivity calculation}

Assuming there is only one sample with a thickness of $\mathrm{t}_{0}$ between the reference planes (no air gaps), the expressions for the reflection coefficient $S_{11}$ and transmission coefficient $S_{21}$ are [26]:

$$
\begin{aligned}
& \mathrm{S}_{11}=\frac{-2\left(\gamma_{0}^{2}+\beta_{0}^{2}\right) \sinh \gamma_{0} t_{0}}{2\left(\gamma_{0}^{2}-\beta_{0}^{2}\right) \sinh \gamma_{0} t_{0}+4 j \gamma_{0} \beta_{0} \cosh \gamma_{0} t_{0}} \\
& \mathrm{~S}_{21}=\frac{4 j \gamma_{0} \beta_{0}}{2\left(\gamma_{0}^{2}-\beta_{0}^{2}\right) \sinh \gamma_{0} t_{0}+4 j \gamma_{0} \beta_{0} \cosh \gamma_{0} t_{0}}
\end{aligned}
$$

where $\gamma_{0}$ is the complex propagation constant, which is given by

$$
\gamma_{0}=j 2 \pi \sqrt{\frac{\varepsilon_{\mathrm{r}}}{\lambda^{2}}-\frac{1}{\lambda_{c}^{2}}}
$$

where $\lambda$ and $\lambda_{\mathrm{c}}=2 \mathrm{a}$ are the free space wavelength and cut-off wavelength, respectively (a is the broader dimension of the waveguide, Fig. 4). $\beta_{0}=2 \pi / \lambda_{g}$ is the phase constant of the transmission line. $\lambda_{g}$ is the guide wavelength of the empty waveguide:

$$
\lambda_{g}=\frac{1}{\sqrt{\frac{1}{\lambda^{2}}-\frac{1}{\lambda_{c}^{2}}}}=\frac{\lambda}{\sqrt{1-\left(\frac{\lambda}{\lambda_{c}}\right)^{2}}}
$$

In the measurement, the sample thickness is shorter than the waveguide offset. In order to fit the $\mathrm{S}_{11}$ and $\mathrm{S}_{21}$ given in Eq. (8), the S-parameters obtained are phase shifted first. The Newton-Raphson approximation method is applied to solve the transcendental equations for the unknown $\gamma_{0}$ : 


$$
\begin{aligned}
& {\left[\mathrm{S}_{11}\right]_{\text {meas }} e^{2 j \beta_{0} \mathrm{~L}_{\text {air }}}-\mathrm{S}_{11}=0} \\
& {\left[\mathrm{~S}_{21}\right]_{\text {meas }} e^{j \beta_{0} \mathrm{~L}_{\text {air }}}-\mathrm{S}_{21}=0}
\end{aligned}
$$

where $\left[\mathrm{S}_{11}\right]_{\text {meas }}$ and $\left[\mathrm{S}_{21}\right]_{\text {meas }}$ are the measured reflection coefficient and transmission coefficient, respectively. $L_{\text {air }}$ is the length of the air gap near Reference plane 1, and the length of the waveguide offset is $34.88 \mathrm{~mm}$. Hence, $\mathrm{L}_{\text {air }}=34.88 \mathrm{~mm}-\mathrm{t}_{0} . \mathrm{t}_{0}$ is $4.20 \mathrm{~mm}$ for the CFRP samples and $2.78 \mathrm{~mm}$ for the neat resin sample.

With the calculated $\gamma_{0}$ value, the complex permittivity $\varepsilon_{\mathrm{r}}$ is given from Eq. (9) as

$$
\varepsilon_{r}=\lambda^{2}\left[\left(\frac{\gamma_{0}}{2 \pi}\right)^{2}+\frac{1}{\lambda_{c}^{2}}\right]
$$

\section{Experimental results}

\subsection{Measured S-parameters}

The measured magnitudes and phases of $\left[\mathrm{S}_{11}\right]_{\text {meas }}$ and $\left[\mathrm{S}_{21}\right]_{\text {meas }}$ are shown in Fig. 5. The good linearity shown in the phase graphs indicates that the measurement is reliable for accurate permittivity calculation. It is seen that more energy is reflected in the parallel case. At $10 \mathrm{GHz}$, the magnitude of $\mathrm{S}_{11}$ in that case is $-0.72 \mathrm{~dB}$, which means that around $85 \%$ of the total power is reflected; in the orthogonal case approximately $57 \%$ is reflected. The magnitude of $\mathrm{S}_{21}$ in the orthogonal case is slightly higher than that in the parallel case.

The fractional loss of the incident power in the samples is evaluated by the energy loss factor, which is the difference between the unitary power and the sum of the squares of $\left|S_{11}\right|$ and $\left|S_{21}\right|$ :

$$
\text { Energy loss factor }(\%)=100 \times\left(1-\left|\mathrm{S}_{11}\right|^{2}-\left|\mathrm{S}_{21}\right|^{2}\right)
$$

The variation of the factor over the frequency band is plotted in Fig. 6. It is found that only around 5 $\%$ energy is absorbed in the neat resin sample, while around $15 \%$ and over $30 \%$ energy is absorbed 
in the parallel and orthogonal cases, respectively. In the orthogonal case, the energy loss factor gradually increases with increasing frequency and reaches a maximum of $48 \%$ at $12 \mathrm{GHz}$.

\subsection{Electric permittivity}

The $S_{21}$ data were used for permittivity calculation, as the transmission measurement was considered more reliable than the reflection one [27,28]. The calculated permittivity values of all the three samples are presented in Fig. 7. Here the CFRP is considered as a homogenous lossy dielectric layer. As illustrated in Fig. 8, three geometric scales of the composite laminate are studied in order to fully understand the nature of the difference in the permittivity: macro-meso scale, microscale and nanoscale. On the macro-meso scale, a number of thin layers of prepregs are stacked with a desired sequence of fibre orientations. On the micro-scale, in a lamina, ideally the fibres do not touch and are completely isolated by the resin. Actually, the fibres are randomly distributed and contact at several points. At the nanoscale level, the electrons in carbon fibres (polycrystalline graphite) are delocalised, i.e., free to move along the planes of carbon atoms.

(a) analysis of the real part of the permittivity: At the microscale level, as illustrated in Fig. 9, due to the presence of the applied EM field, carbon fibres and dielectric epoxy make up a series of capacitors. The capacitance always exists when the incident field is applied at an arbitrary angle. Hence a similar energy storage capability is reflected in the real permittivity. And that permittivity is two orders of magnitude larger than that of the neat resin as would be expected. $\varepsilon_{\mathrm{r}}^{\prime}$ of the parallel and orthogonal cases gradually decreases with increasing frequency, which agrees with the wellestablished relationship between the impedance of capacitors $Z_{c}$ and the frequency, $Z_{c}=1 / j \omega C$, where $\mathrm{C}$ is the capacitance.

(b) analysis of the imaginary part of the permittivity: $\varepsilon_{\mathrm{r}}^{\prime \prime}$ of the orthogonal case is approximately half of that for the parallel case. As the epoxy resin is a polar dielectric material [29], only the dipolar relaxation exists. The lower $\varepsilon_{\mathrm{r}}^{\prime \prime}$ of the epoxy indicates that the epoxy itself does not contribute much to 
the high loss of the composite, and the primary loss is associated with the carbon fibres. At the nanoscale level, when the electric field is parallel to the fibres, the electrons move freely along the whole length of the fibres. In the orthogonal case, some electrons travel via the contact points between fibres, while the others are trapped at the interfaces between the fibres and resin (MaxwellWagner (interfacial) polarisation [30]), which leads to a relatively lower conductivity. This relationship between the imaginary permittivity (Fig. 7 (b)) and the corresponding energy absorbed (Fig. 6) in the two composites cases agrees with the trend demonstrated in Fig. 1.

The effect of the calibration error on the accuracy of the calculated permittivity is investigated. As listed in Table 1, four combinations with the magnitude and phase of $S_{21}$ added or subtracted by the calibration errors of attenuation (i.e., $\pm 0.1 \mathrm{~dB}$ ) and phase shift (i.e., $\pm 0.5^{\circ}$ ) are used.

Taking the CFRP-parallel sample as an example, the permittivity values for the combination cases together with the data in Fig. 7 ("Reference”) are presented in Fig. 10, where the curves are closely spaced. By comparing the permittivity differences between the four combinations, the measurement uncertainties are obtained. The permittivity values with measurement uncertainties at five frequencies are listed in Table 2. It is seen that the calibration errors only have a significant effect on the imaginary part of the permittivity of the neat resin.

The loss tangent values of the three samples over 8-12 GHz is shown in Fig. 11. The loss tangent of the resin is within 0.04 , and the loss tangent for the parallel case is consistently larger than that for the orthogonal case.

\subsection{Electrical conductivity}

Employing Eq. (5), the microwave effective electrical conductivity of each sample is calculated. As given in Fig. 12, due to the conductive fibres, the conductivities of both CFRP samples are significantly increased compared with the neat resin. It is also implied that the conductivity is not considerably frequency dependent. The average effective conductivities for the parallel and orthogonal cases over 8-12 GHz are $37.2 \mathrm{~S} / \mathrm{m}$ and $17 \mathrm{~S} / \mathrm{m}$, respectively. 


\subsection{Signal penetration}

The penetration depths calculated using the permittivity values (Fig. 7) and Eq. (6) are presented in Fig. 13. It is clear that the microwave signal can easily pass through the resin. However, the signal is strongly attenuated in the two CFRP samples. For better signal penetration, the electric field should be placed orthogonal to the fibres, or a lower frequency can be adopted. If the material is assumed metallic and Eq. (7) is selected for calculation, in order to match with the results using Eq. (6), conductivities of $10 \mathrm{~S} / \mathrm{m}$ and $2.4 \mathrm{~S} / \mathrm{m}$ are used for the parallel and orthogonal cases, respectively. It is seen that the equivalent metallic conductivity is smaller than the effective conductivity presented in Section 4.3, which agrees with the definition of the effective conductivity (Eq. (5)).

\subsection{Microwave absorption}

Here the absorption performance is further investigated with a widely used model for assessing the absorption properties of a dielectric, as illustrated in Fig. 14, where a plane wave is normally incident from free space onto the surface of a single dielectric layer backed by Perfect Electric Conductor (PEC). The transversal dimensions of the layer are assumed infinite. The reflection coefficient $\mathrm{S}_{11}$ can be evaluated using Eq. (14):

$$
\begin{gathered}
\mathrm{S}_{11}(\mathrm{~dB})=20 \log _{10}\left|\mathrm{~S}_{11}\right| \\
\mathrm{S}_{11}=\frac{z_{\text {in }}-1}{z_{\text {in }}+1} \\
z_{\text {in }}=\sqrt{\frac{\mu_{\mathrm{r}}}{\varepsilon_{\mathrm{r}}}} \tanh \left[j \frac{2 \pi f t}{c} \sqrt{\mu_{\mathrm{r}} \varepsilon_{\mathrm{r}}}\right]
\end{gathered}
$$

where $\mathrm{z}_{\text {in }}$ is the normalised input impedance at the free space-material interface. $\left|\mu_{r}\right|$ and $\left|\varepsilon_{r}\right|$ are the moduli of the relative permeability $\mu_{r}$ and relative permittivity $\varepsilon_{\mathrm{r}}$, respectively. 
To achieve an optimal response, the sample thickness $t$ can be designed to be an odd multiple of the quarter wavelength in the material, so that the incident and reflected waves in the material are out of phase, which results in no reflection at the interface [31].

$$
\begin{gathered}
t=n \frac{\lambda_{m}}{4}(\mathrm{n}=1,3,5, \cdots) \\
\lambda_{m}=\frac{\lambda_{d}}{\sqrt{\left(\left|\mu_{r}\right|\left|\varepsilon_{r}\right|\right)}}
\end{gathered}
$$

where $\lambda_{\mathrm{d}}=c / f_{\mathrm{d}}$ is the free space wavelength at a design frequency $f_{\mathrm{d}}$.

Here $f_{\mathrm{d}}$ is set to $10 \mathrm{GHz}$, and the corresponding permittivity values are used for determination of the optimal sample thicknesses, which are listed in Table 3. The reflection coefficients simulated over X-band are shown in Fig. 15. The minimum reflection occurs in the orthogonal case with approximately six plies (typical ply thickness $0.125 \mathrm{~mm}$ ), where a maximum of $84 \%$ power absorption is found. It is also demonstrated that with a lower conductivity better microwave absorption could be obtained.

\section{Concluding remarks}

A carbon fibre composite laminate has been characterised over $\mathrm{X}$ band (8-12 GHz) using the transmission line method. By analysing the permittivity at three geometric scales, the mechanism behind the interaction between the composites and the electromagnetic field has been fully understood. It has been revealed that on the macro-meso scale the fibre orientation with respect to the incident electric field has a significant effect on the electromagnetic responses. More energy is reflected when the electric field vector is made parallel to the fibres. The real permittivity $\varepsilon_{\mathrm{r}}^{\prime}$ is related to the microscale, where the capacitive effect caused by the conductive carbon fibres and dielectric epoxy exists. $\varepsilon_{\mathrm{r}}^{\prime}$ of the parallel case is close to that of the orthogonal case, as similar capability of 
energy storage is offered. And the imaginary permittivity $\varepsilon_{\mathrm{r}}^{\prime \prime}$ is more associated with the nanoscale level. Over the frequency range investigated, the imaginary permittivity $\varepsilon_{\mathrm{r}}^{\prime \prime}$ of the parallel case is approximately twice that of the orthogonal case. The difference is due to the distinct response of the free charges in the polycrystalline graphite structures with respect to the electric field.

The conductivity results show that carbon fibre composites behave more like a lossy conducting material. From the calculation of the penetration depth and energy loss factor, it is revealed that the incident signal is highly attenuated within the composites. In terms of the energy loss factor, there are of twice difference between the two CFRP cases, both of which are one order of magnitude larger than the neat resin case. More energy is absorbed in the orthogonal case. In addition, the microwave absorbing capability of CFRP has been evaluated with a practical setup, where a material layer is backed by a conductor plate. With optimal design a maximum of $84 \%$ power absorption is achieved, which suggests that the continuous carbon fibre reinforced composites can be used as an alternative microwave absorbing material other than the carbon-based nanocomposites.

On the non-destructive detection aspect, for surface damage the electric field should be parallel to the fibres, so that the signal is more sensitive to the changes in the standoff distance and local permittivity distribution [32]. For internal damage the EM field shall be orthogonal to the fibres in order to obtain better signal penetration. The permittivity results presented here could be used as a reference input for simulation of microwave curing, heating and electromagnetic shielding of polymer composites employed in aerospace and other industries.

\section{Acknowledgements}

This work was funded by Dean's Doctoral Scholar Award, Faculty of Science and Engineering, The University of Manchester. The first author gratefully acknowledges the support of Dr. Edward Pullicino and Dr. Ying Wang from School of Materials for the assistance in the sample preparation. Special thanks to Prof. Robin Sloan for useful discussions. 


\section{References}

[1] Soutis C. Carbon fiber reinforced plastics in aircraft construction. Mater Sci Eng A 2005;412:171-6.

[2] Xu Z, Gao C. Graphene fiber: a new trend in carbon fibers. Mater Today 2015;18:480-92.

[3] Mallick PK. Fiber-reinforced composites : materials, manufacturing, and design. 3rd ed. CRC Press; 2007.

[4] Kaiser JH. Reflection and Absorption of Microwaves (2-100 GHz) by Carbon-FiberReinforced Composite Surfaces. Res Nondestruct Eval 1994;5:275-83.

[5] Cordill BD, Seguin SA, Ewing MS. Shielding effectiveness of composite and aluminum aircraft, model and measurement comparison. 2011 IEEE Int. Instrum. Meas. Technol. Conf., IEEE; 2011, p. 1-5.

[6] Riley EJ, Lenzing EH, Narayanan RM. Characterization of radar cross section of carbon fiber composite materials. In: Ranney KI, Doerry A, Gilbreath GC, Hawley CT, editors., 2015, p. 946103.

[7] Krupka J. Frequency domain complex permittivity measurements at microwave frequencies. Meas Sci Technol 2006;17:R55-70.

[8] Micheli D, Apollo C, Pastore R, Marchetti M. X-Band microwave characterization of carbonbased nanocomposite material, absorption capability comparison and RAS design simulation. Compos Sci Technol 2010;70:400-9.

[9] Oh J-H, Oh K-S, Kim C-G, Hong C-S. Design of radar absorbing structures using glass/epoxy composite containing carbon black in X-band frequency ranges. Compos Part B Eng 2004;35:49-56.

[10] Kim J-B. Broadband radar absorbing structures of carbon nanocomposites. Adv Compos Mater 2012;21:333-44. 
[11] Micheli D, Vricella A, Pastore R, Marchetti M. Synthesis and electromagnetic characterization of frequency selective radar absorbing materials using carbon nanopowders. Carbon N Y $2014 ; 77: 756-74$.

[12] Verma M, Chauhan SS, Dhawan SK, Choudhary V. Graphene nanoplatelets/carbon nanotubes/polyurethane composites as efficient shield against electromagnetic polluting radiations. Compos Part B Eng 2017;120:118-27.

[13] Yoon SS, Lee JW, Lee TK, Roh JH, Kim HI, Yi DW. Conductivity Evaluation of a Newly Proposed Material for a SAR Reflector Antenna. J Electromagn Eng Sci 2014;14:293-8.

[14] Yoon SS, Lee JW, Lee T-K, Roh JH. Insensitivity Characteristics in the Dual Polarization of Deployable CFRP Reflector Antennas for SAR. IEEE Trans Antennas Propag 2018;66:88-95.

[15] Galehdar A, Rowe WST, Ghorbani K, Callus PJ, John S, Wang CH. The effect of ply orientation on the performance of antennas in or on carbon fiber composites. Prog Electromagn Res 2011;116:123-36.

[16] Riley EJ, Lenzing EH, Narayanan RM. Characterization of carbon fiber composite materials for RF applications. In: Ranney KI, Doerry A, editors. SPIE Def. + Secur., International Society for Optics and Photonics; 2014, p. 907704.

[17] Bull DA, Jackson GA, Smithers BW. Radio frequency characteristics of carbon fibre composite materials. Radio Electron Eng 1981;51:505.

[18] Ashcroft NW, Mermin ND. Solid state physics. Brooks Cole; 1976.

[19] Salski B, Gwarek W, Korpas P. Electromagnetic inspection of carbon-fiber-reinforced polymer composites with coupled spiral inductors. IEEE Trans Microw Theory Tech $2014 ; 62: 1535-44$.

[20] Marion J, Hornyak W. Physics for science and engineering. CBS college publishing; 1982.

[21] Lee S-E, Park K-Y, Oh K-S, Kim C-G. The use of carbon/dielectric fiber woven fabrics as filters for electromagnetic radiation. Carbon N Y 2009;47:1896-904. 
[22] Pozar D. Microwave engineering. Fourth edi. New York: John Wiley \& Sons; 2011.

[23] Hippel V, Robert A. Dielectric materials and applications. 2nd ed. New York: Artech House; 1995.

[24] Thostenson ET, Chou T-W. Microwave processing: fundamentals and applications. Compos Part A Appl Sci Manuf 1999;30:1055-71.

[25] Dauda B, Oyadiji SO, Potluri P. Characterising mechanical properties of braided and woven textile composite beams. Appl Compos Mater 2008;16:15-31.

[26] Tischer FJ. Measurement of the wave-propagation properties of plasma in the microwave region. IRE Trans Instrum 1960;I-9:167-71.

[27] Haigh AD, Thompson F, Gibson AAP, Campbell GM, Fang C. Complex permittivity of liquid and granular materials using waveguide cells. Subsurf Sens Technol Appl 2001;2:425-34.

[28] Li Z, Haigh A, Soutis C, Gibson A, Sloan R. Dielectric constant of a three-dimensional woven glass fibre composite: Analysis and measurement. Compos Struct 2017;180:853-61.

[29] Maity P, Kasisomayajula S, Parameswaran V, Basu S, Gupta N. Improvement in surface degradation properties of polymer composites due to pre-processed nanometric alumina fillers. IEEE Trans Dielectr Electr Insul 2008;15:63-72.

[30] Kremer F, Schönhals A, editors. Broadband Dielectric Spectroscopy. Berlin, Heidelberg: Springer Berlin Heidelberg; 2003.

[31] Deng L, Han M. Microwave absorbing performances of multiwalled carbon nanotube composites with negative permeability. Appl Phys Lett 2007;91:023119.

[32] Li Z, Haigh A, Soutis C, Gibson A, Sloan R. Applications of microwave techniques for aerospace composites. 2017 IEEE Int. Conf. Microwaves, Antennas, Commun. Electron. Syst., Tel Aviv,Israel: IEEE; 2017, p. 1-4. 


\section{Figure captions}

Fig. 1. Relationship between the dielectric loss factor and the microwave power absorption capability (adapted from [24]).

Fig. 2. Layup of the $T 300$ preform before the resin infusion process.

Fig. 3. Experimental setup for microwave characterisation of a CFRP sample: (a) setup of the transmission line method; (b) location of the sample within the waveguide cell.

Fig. 4. Diagrams showing the fibre direction of the composite sample with respect to the electric field vector inside the waveguide: (a) parallel case; (b) orthogonal case.

Fig. 5. Measured $S_{11}$ and $S_{21}$ in the form of magnitude and phase over 8-12 GHz: (a) magnitudes of $\mathrm{S}_{11}$; (b) phases of $\mathrm{S}_{11}$; (c) magnitudes of $\mathrm{S}_{21}$; (d) phases of $\mathrm{S}_{21}$.

Fig. 6. Variation of the energy loss factor with respect to the frequency for the three test samples. Fig. 7. Relative permittivity of the neat resin and two CFRP samples with fibres in perpendicular directions: (a) $\varepsilon_{\mathrm{r}}^{\prime}$; (b) $\varepsilon_{\mathrm{r}}^{\prime \prime}$.

Fig. 8. Three geometric scales used for study of the complex permittivity of a composite laminate. Fig. 9. Schematic diagram of the equivalent circuit model of a composite lamina when interacting with the incident electromagnetic field.

Fig. 10. Calculated permittivity for the four cases considering the calibration errors: (a) $\varepsilon_{\mathrm{r}}^{\prime}$; (b) $\varepsilon_{\mathrm{r}}^{\prime \prime}$.

Fig. 11. The loss tangent values of the neat resin and two CFRP samples over 8-12 GHz.

Fig. 12. The conductivity values of the neat resin and two CFRP samples over 8-12 GHz.

Fig. 13. The penetration depths for the neat resin and two CFRP samples with fibres in perpendicular directions over 8-12 GHz.

Fig. 14. Model of a single sample layer backed by PEC with a normally incident plane wave.

Fig. 15. Reflection coefficients for the cases where the material layer with an optimal thickness is backed by PEC at normal incidence in free space. 


\section{Tables}

Table 1 Four combinations considering the effect of the calibration errors on the accuracy of the calculated permittivity

\begin{tabular}{lcccc}
\hline & Combination I & Combination II & Combination III & Combination IV \\
\hline Magnitude error (dB) & +0.1 & +0.1 & -0.1 & -0.1 \\
Phase error (degree) & +0.5 & -0.5 & +0.5 & -0.5 \\
\hline
\end{tabular}

Table 2 Relative permittivity of the neat resin and CFRP samples with measurement uncertainties

\begin{tabular}{|c|c|c|c|c|c|c|}
\hline & & $8 \mathrm{GHz}$ & $9 \mathrm{GHz}$ & $10 \mathrm{GHz}$ & $11 \mathrm{GHz}$ & $12 \mathrm{GHz}$ \\
\hline \multirow{2}{*}{ Neat resin } & $\varepsilon_{r}^{\prime}$ & $2.95 \pm 0.05$ & $2.92 \pm 0.05$ & $2.90 \pm 0.05$ & $2.91 \pm 0.05$ & $2.89 \pm 0.05$ \\
\hline & $\varepsilon_{r}^{\prime \prime}$ & $0.09 \pm 0.05$ & $0.10 \pm 0.05$ & $0.10 \pm 0.06$ & $0.11 \pm 0.06$ & $0.10 \pm 0.06$ \\
\hline \multirow{2}{*}{ CFRP-parallel } & $\varepsilon_{r}^{\prime}$ & $169.65 \pm 0.72$ & $134.18 \pm 1.46$ & $101.26 \pm 0.88$ & $77.85 \pm 0.23$ & $70.91 \pm 0.74$ \\
\hline & $\varepsilon_{r}^{\prime \prime}$ & $65.19 \pm 0.66$ & $78.27 \pm 0.44$ & $73.09 \pm 0.32$ & $59.97 \pm 0.31$ & $49.24 \pm 0.22$ \\
\hline \multirow{2}{*}{ CFRP-orthogonal } & $\varepsilon_{r}^{\prime}$ & $148.05 \pm 0.35$ & $124.16 \pm 0.25$ & $106.78 \pm 0.20$ & $94.15 \pm 0.17$ & $84.81 \pm 0.15$ \\
\hline & $\varepsilon_{r}^{\prime \prime}$ & $35.08 \pm 0.42$ & $33.12 \pm 0.31$ & $30.66 \pm 0.25$ & $28.55 \pm 0.22$ & $27.30 \pm 0.20$ \\
\hline
\end{tabular}

Table 3 Designed sample thickness and the corresponding microwave absorption performance Sample thickness $(t, \mathrm{~mm}) \quad$ Minimum $\mathrm{S}_{11}(\mathrm{~dB}) \quad$ Power absorbed (\%)

\begin{tabular}{cccc}
\hline Neat resin & 4.40 & -1.10 & 22.29 \\
CFRP-parallel & 0.67 & -4.14 & 61.45 \\
CFRP-orthogonal & 0.71 & -8.08 & 84.43 \\
\hline
\end{tabular}




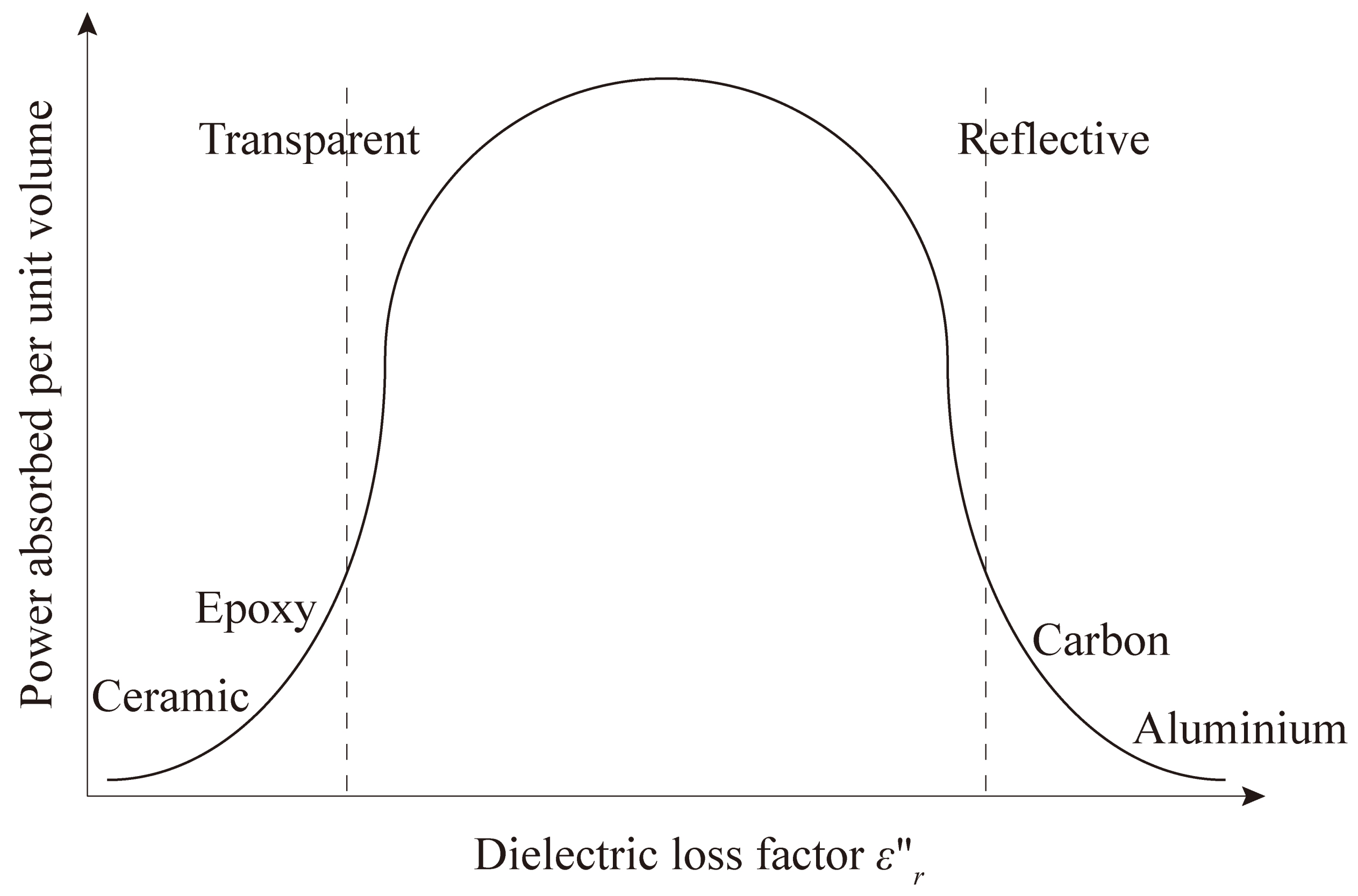




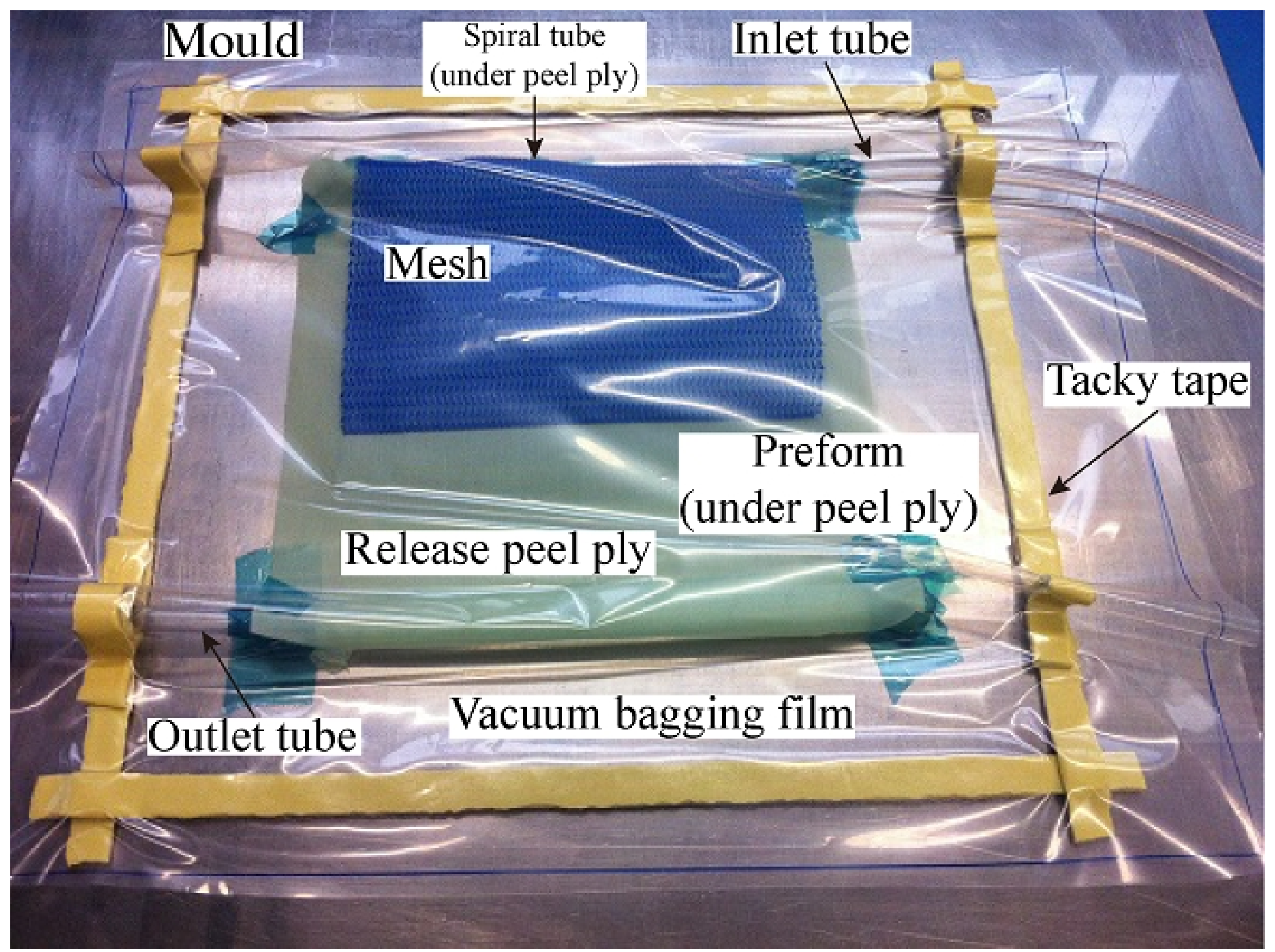




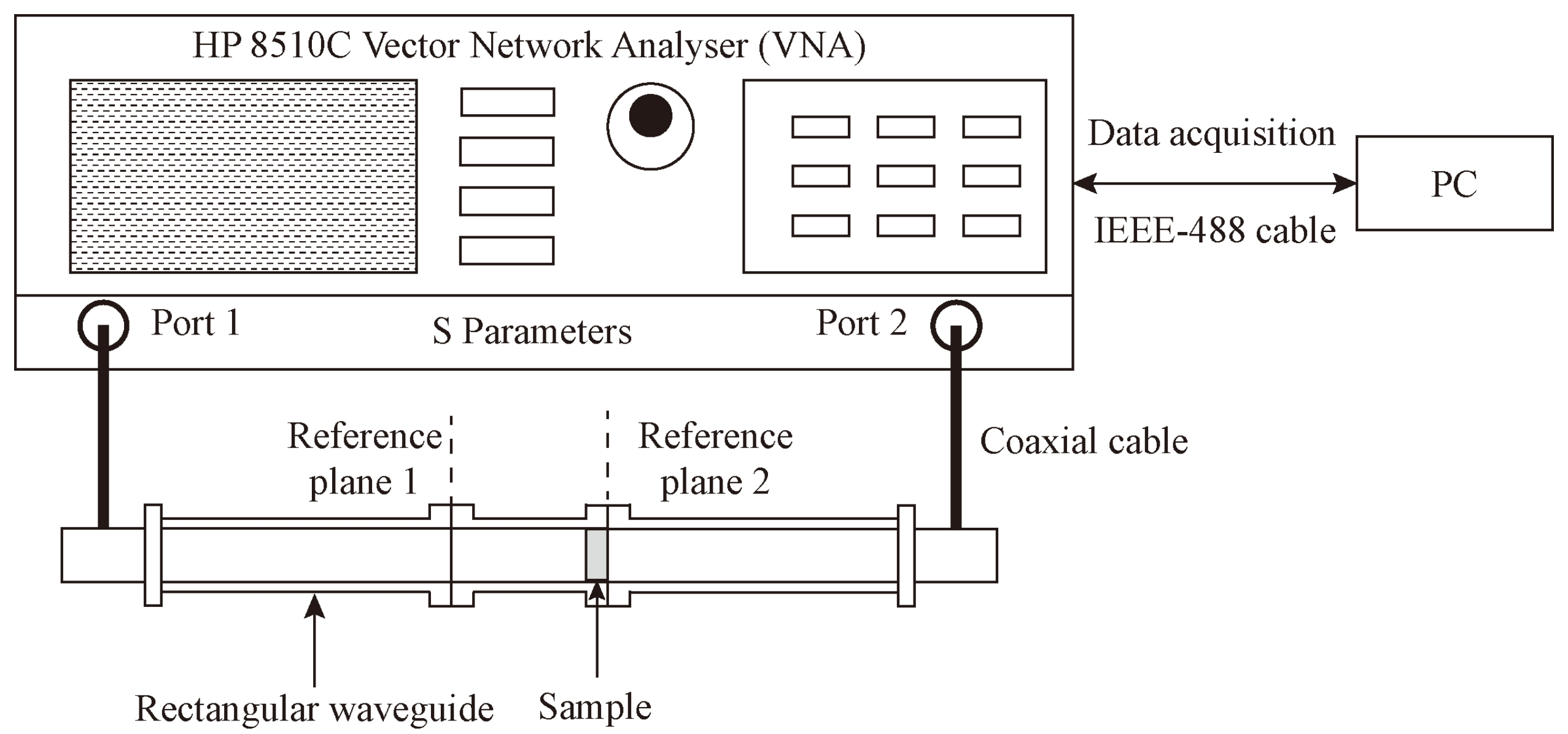




\section{Reference}

| plane 1

\section{Air gap}

Reference, plane 2 ।

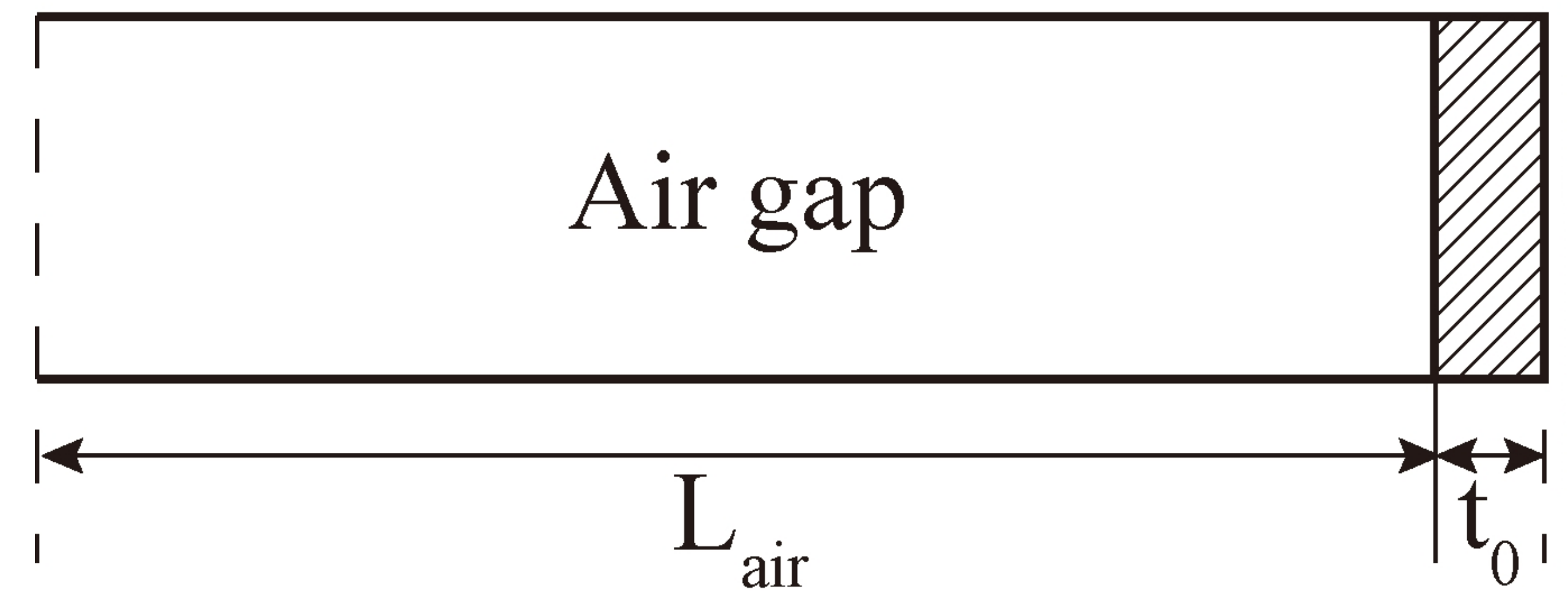


Electric field

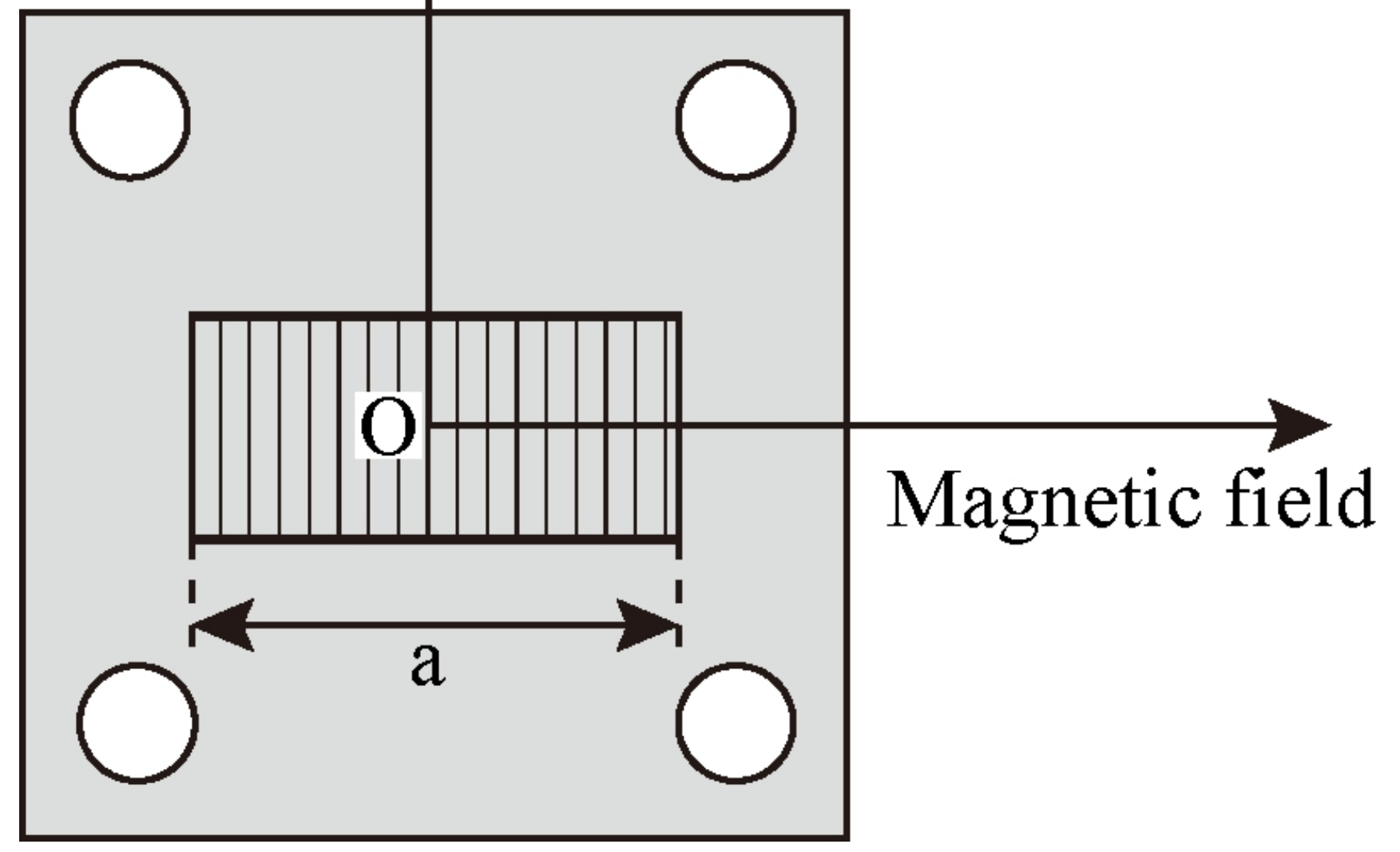


Electric field

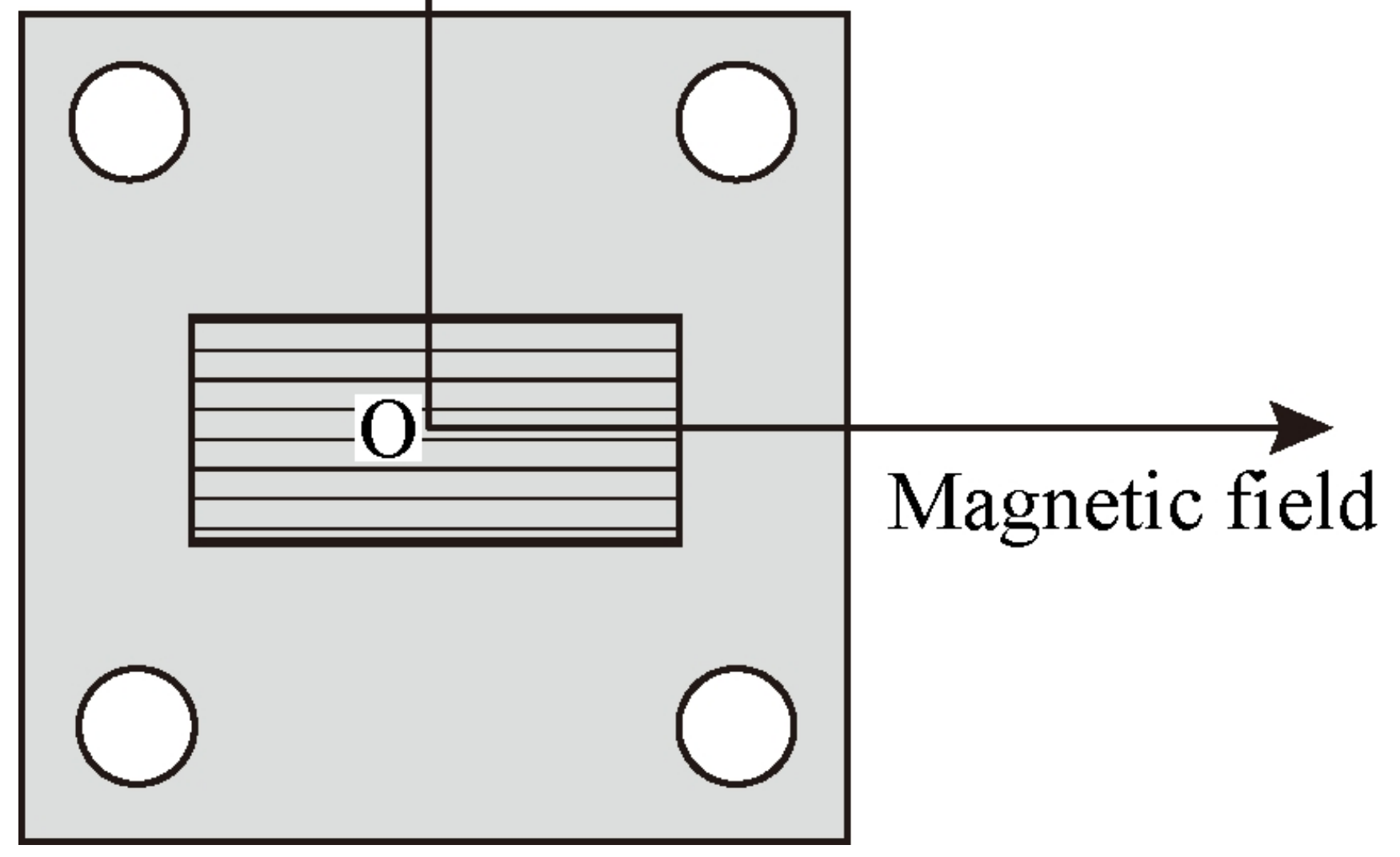




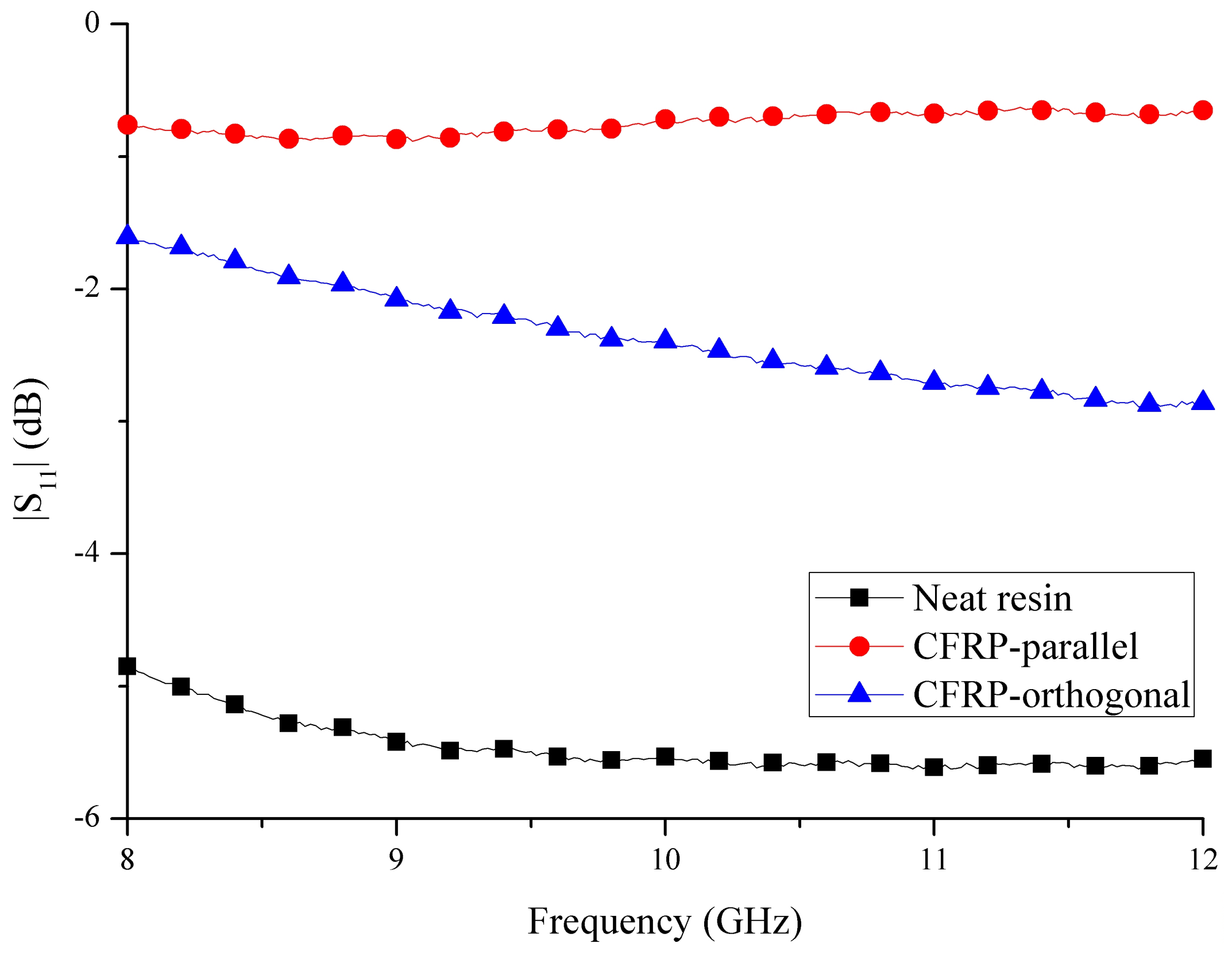




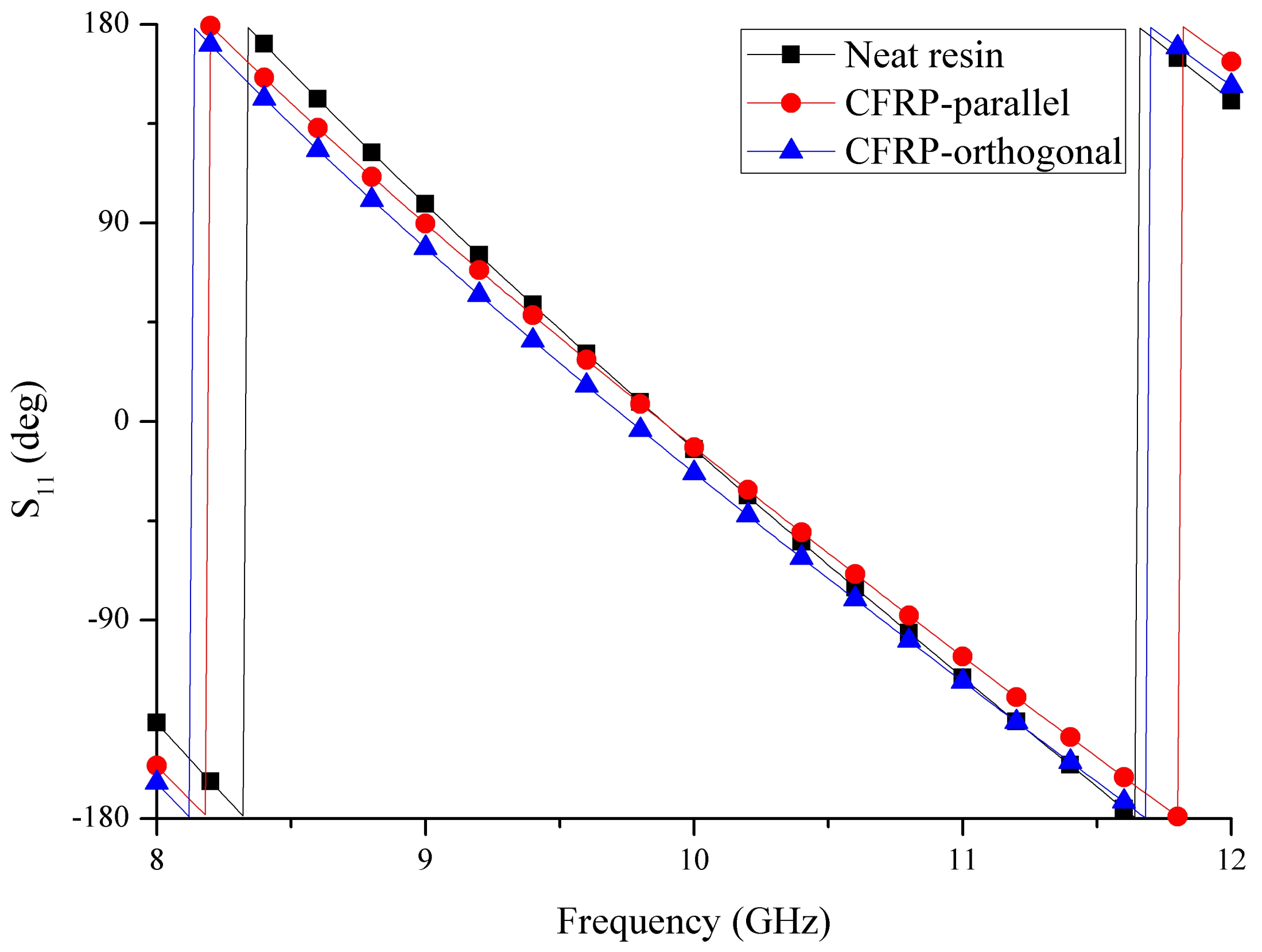




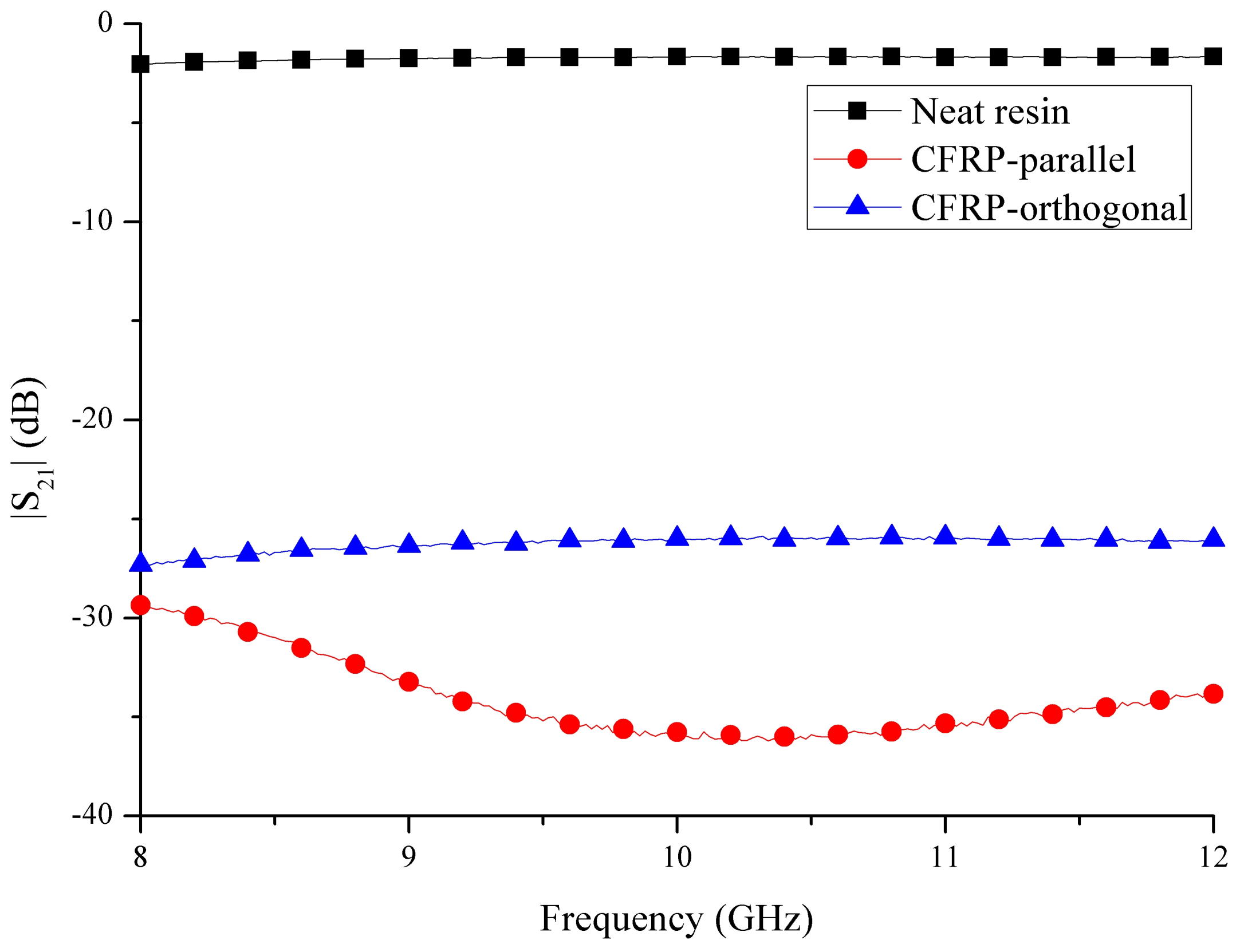




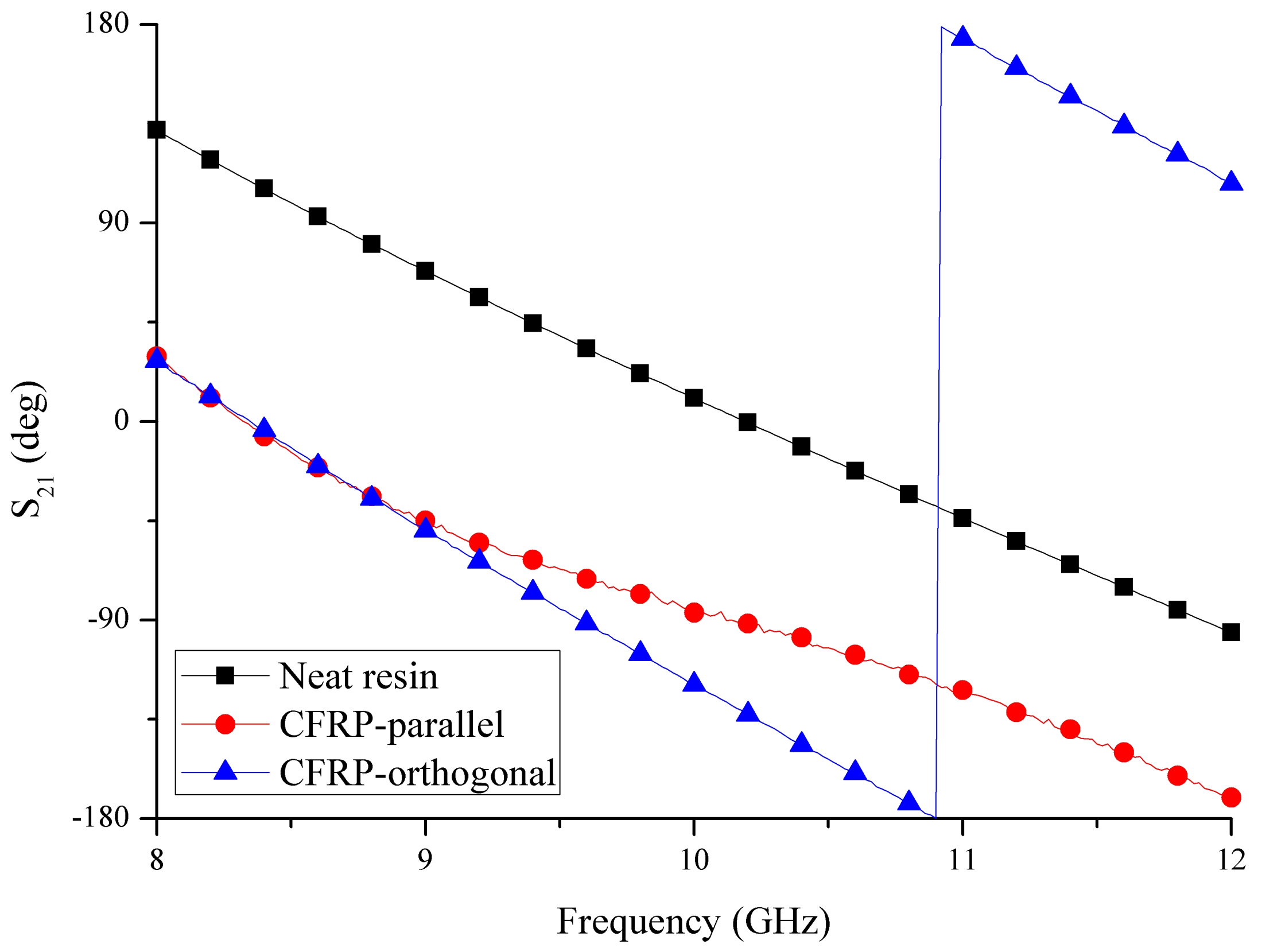




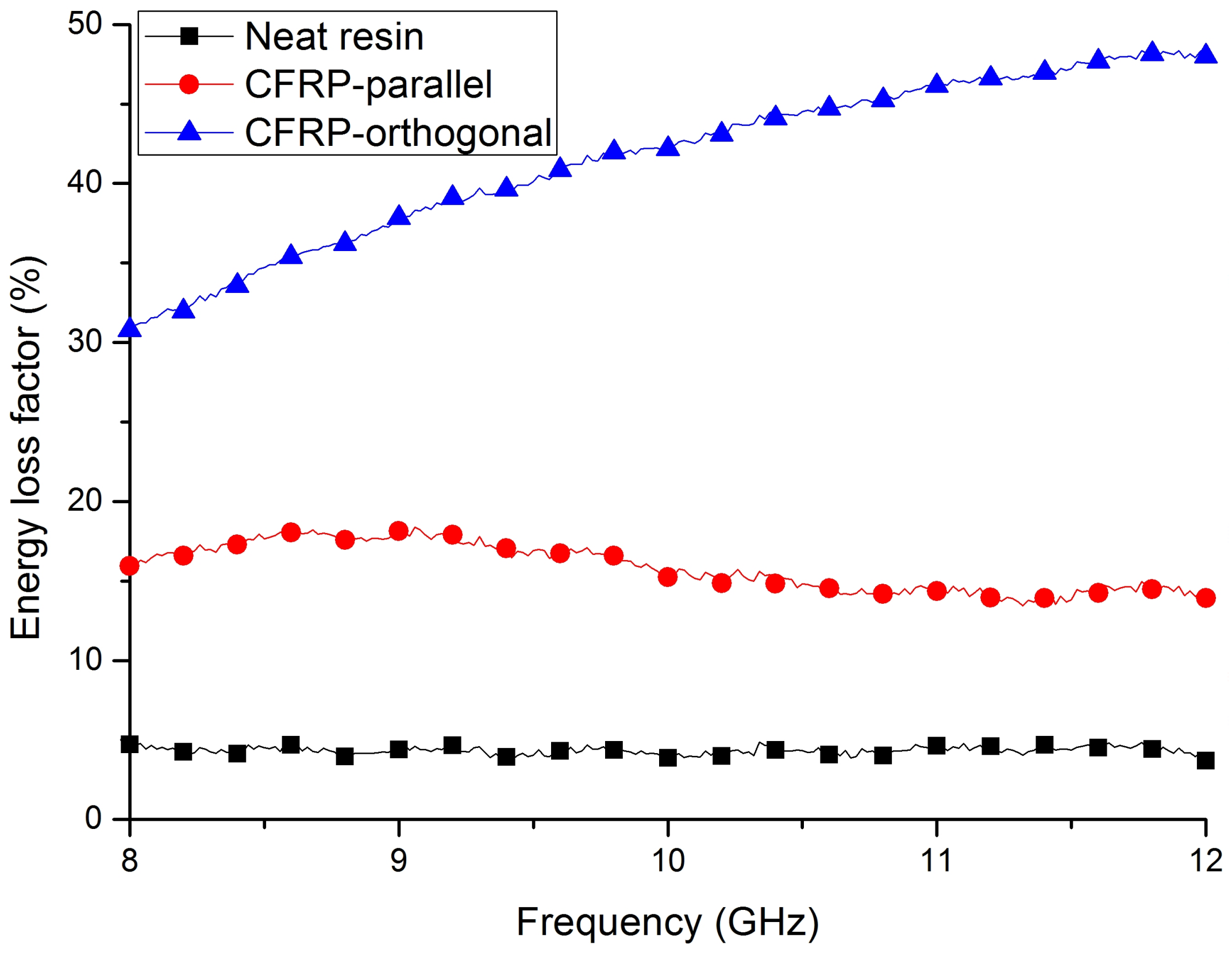




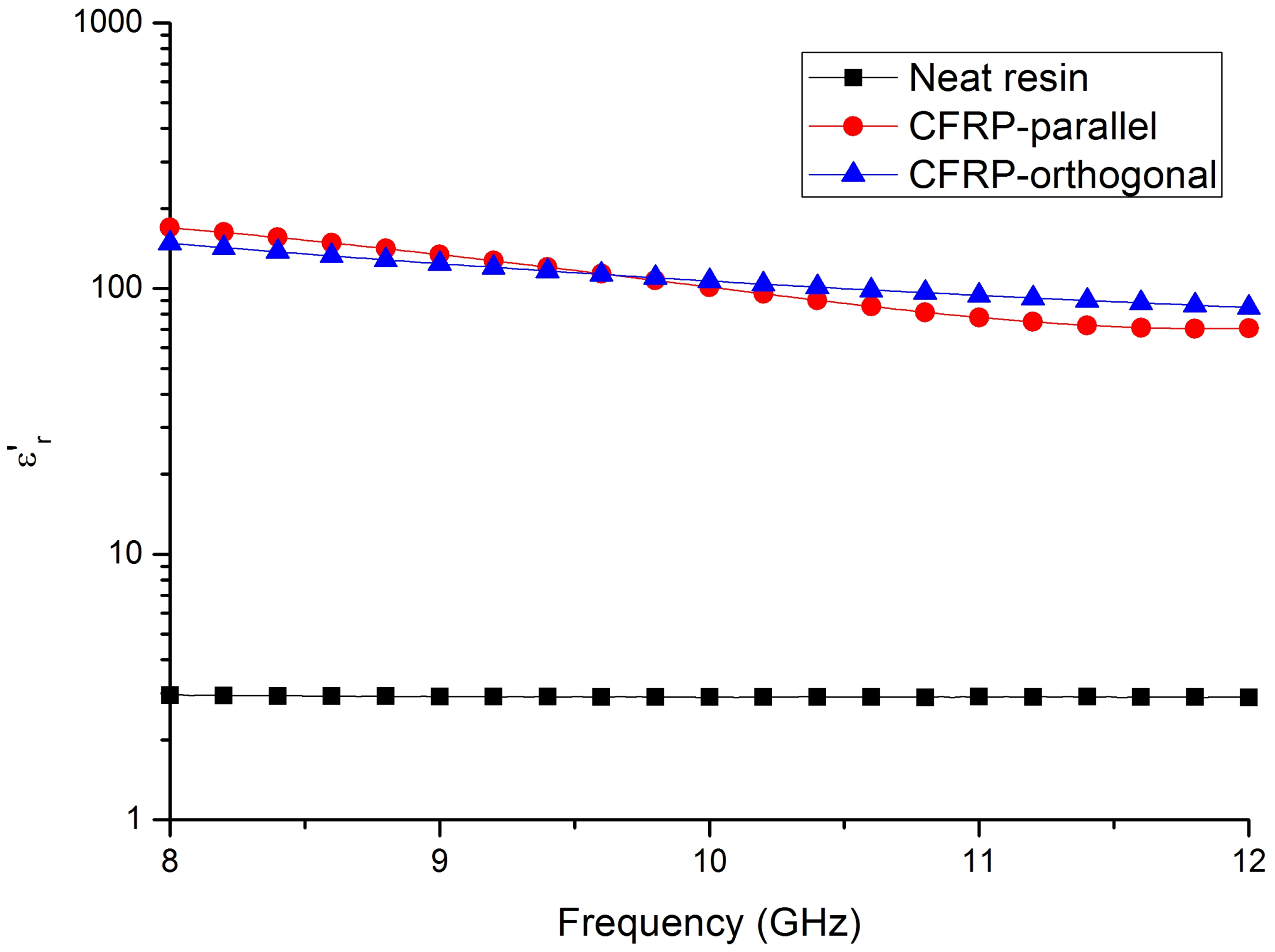




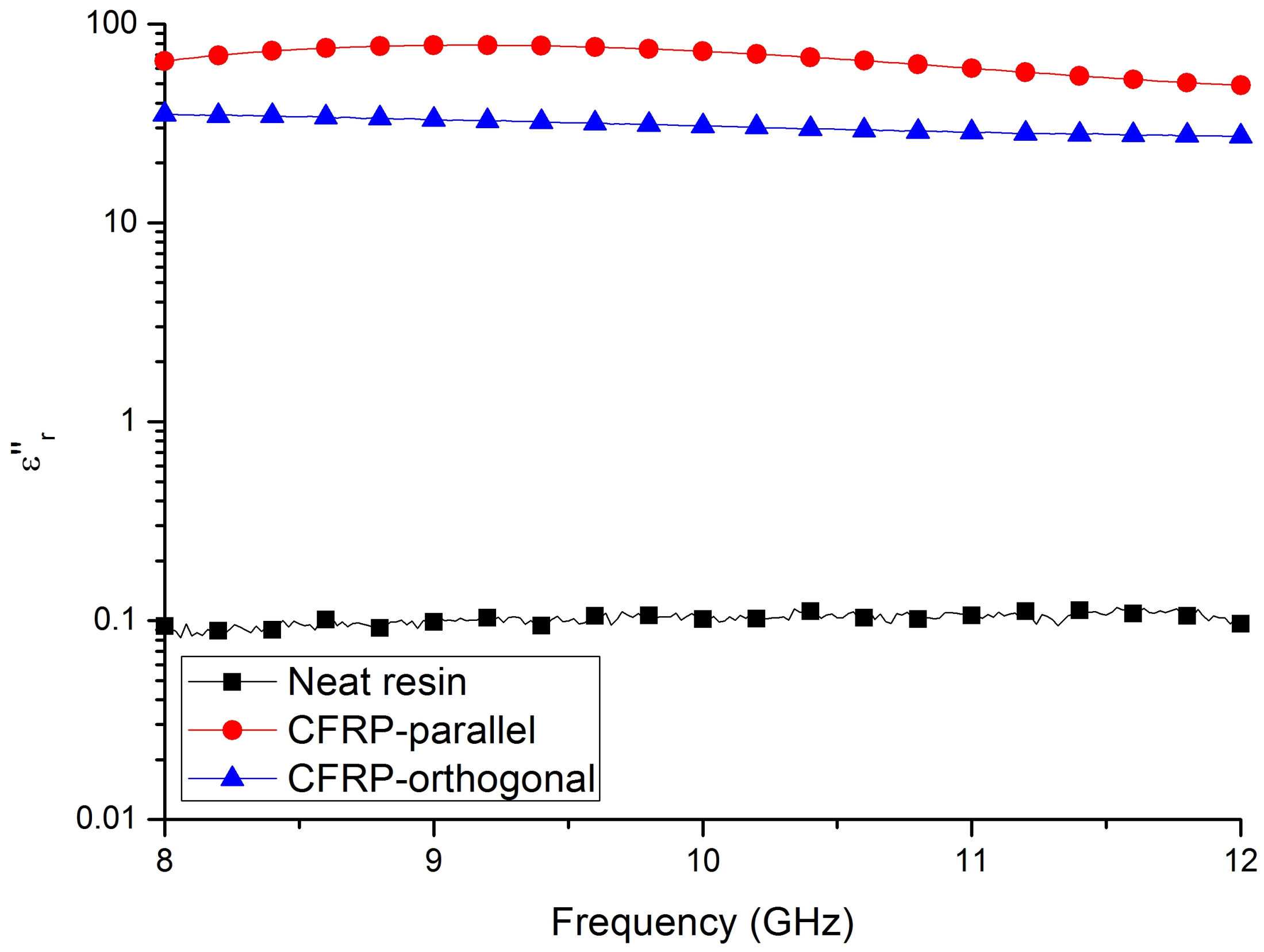




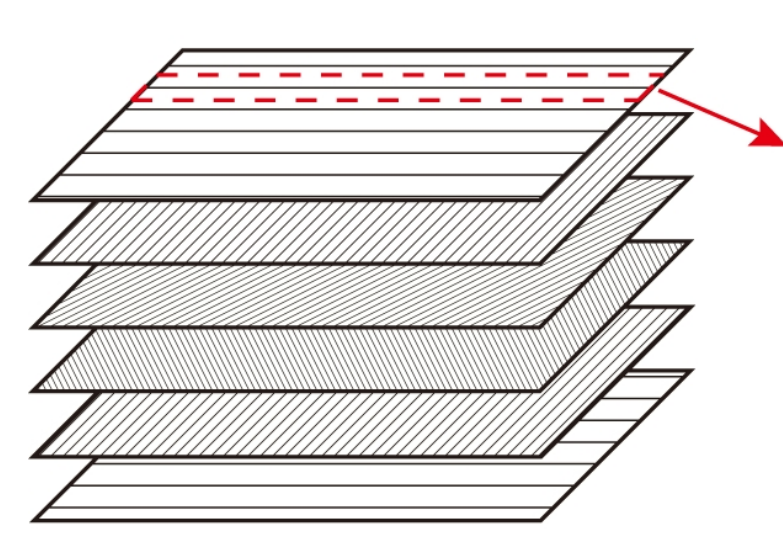

Macro-meso scale

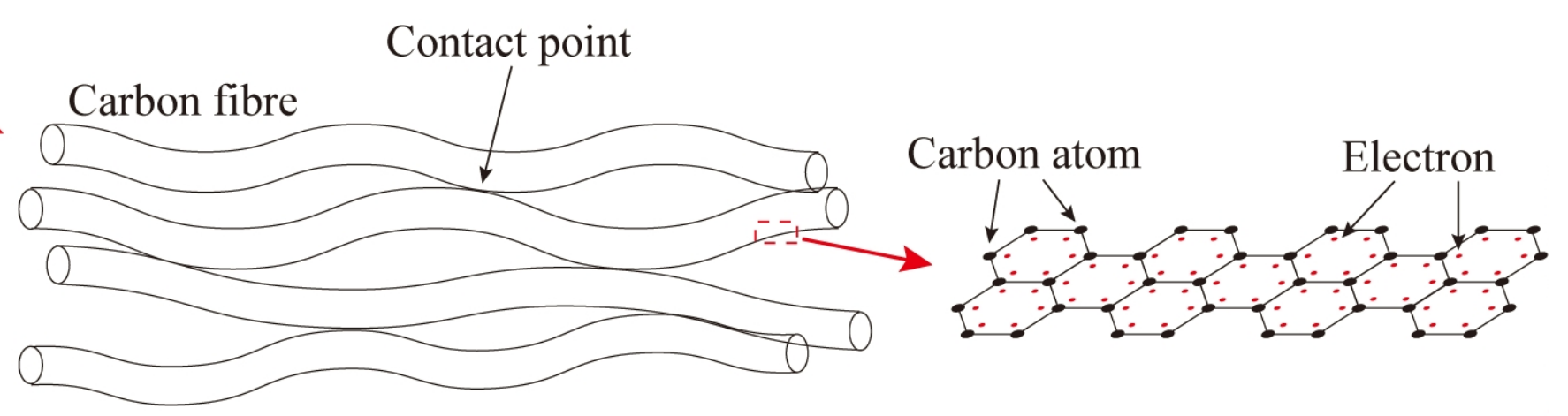

Microscale
Nanoscale 


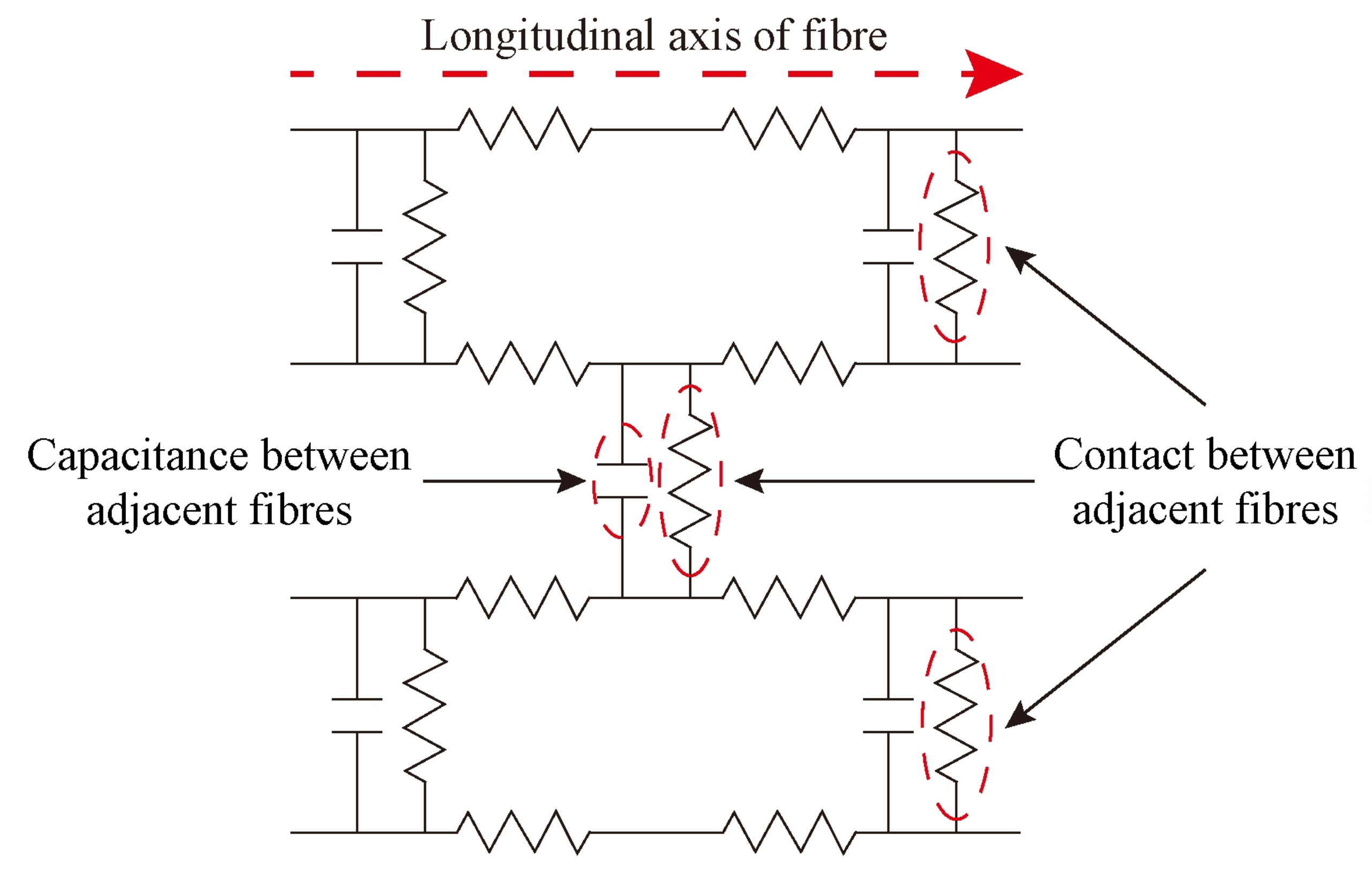




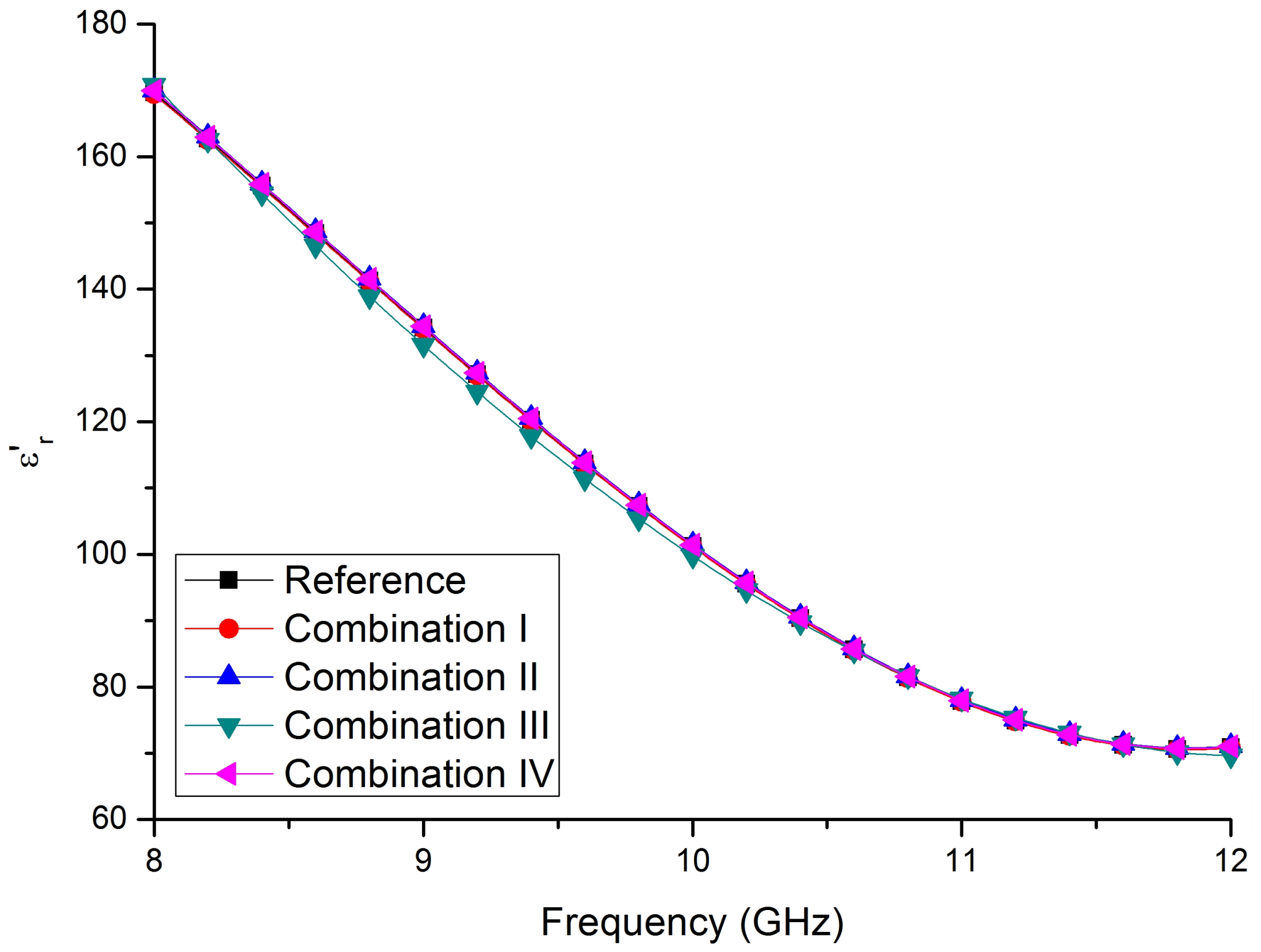




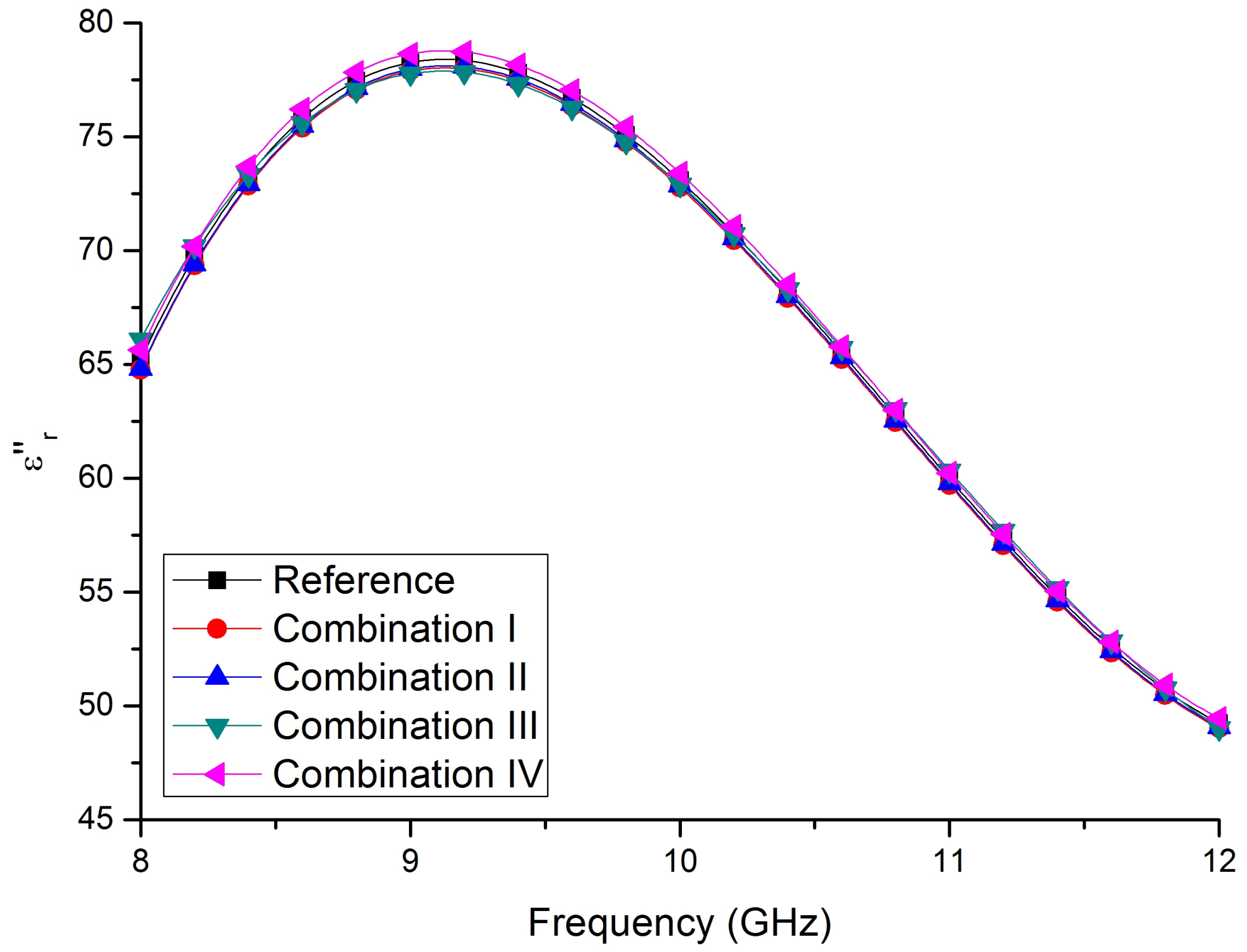




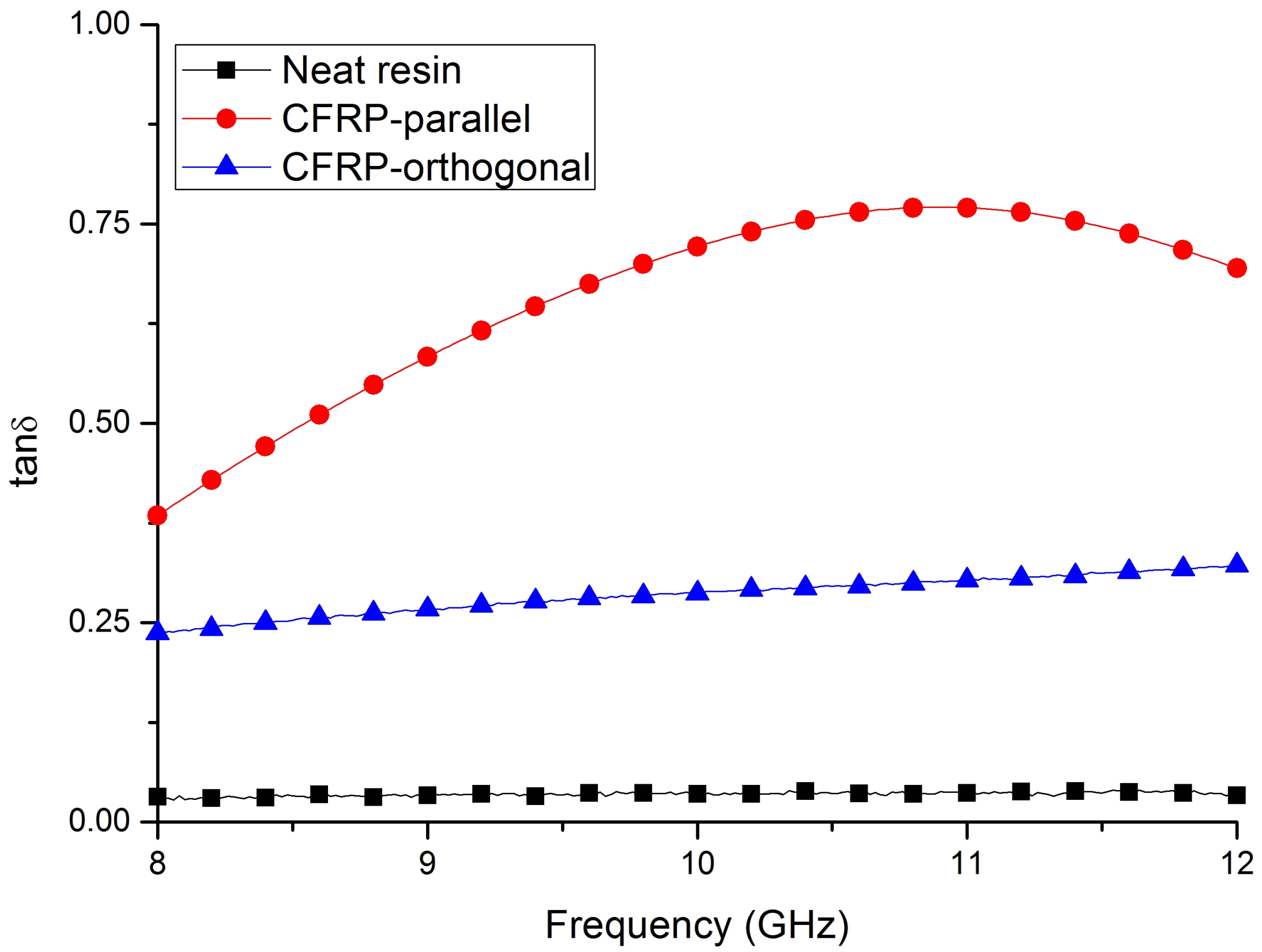




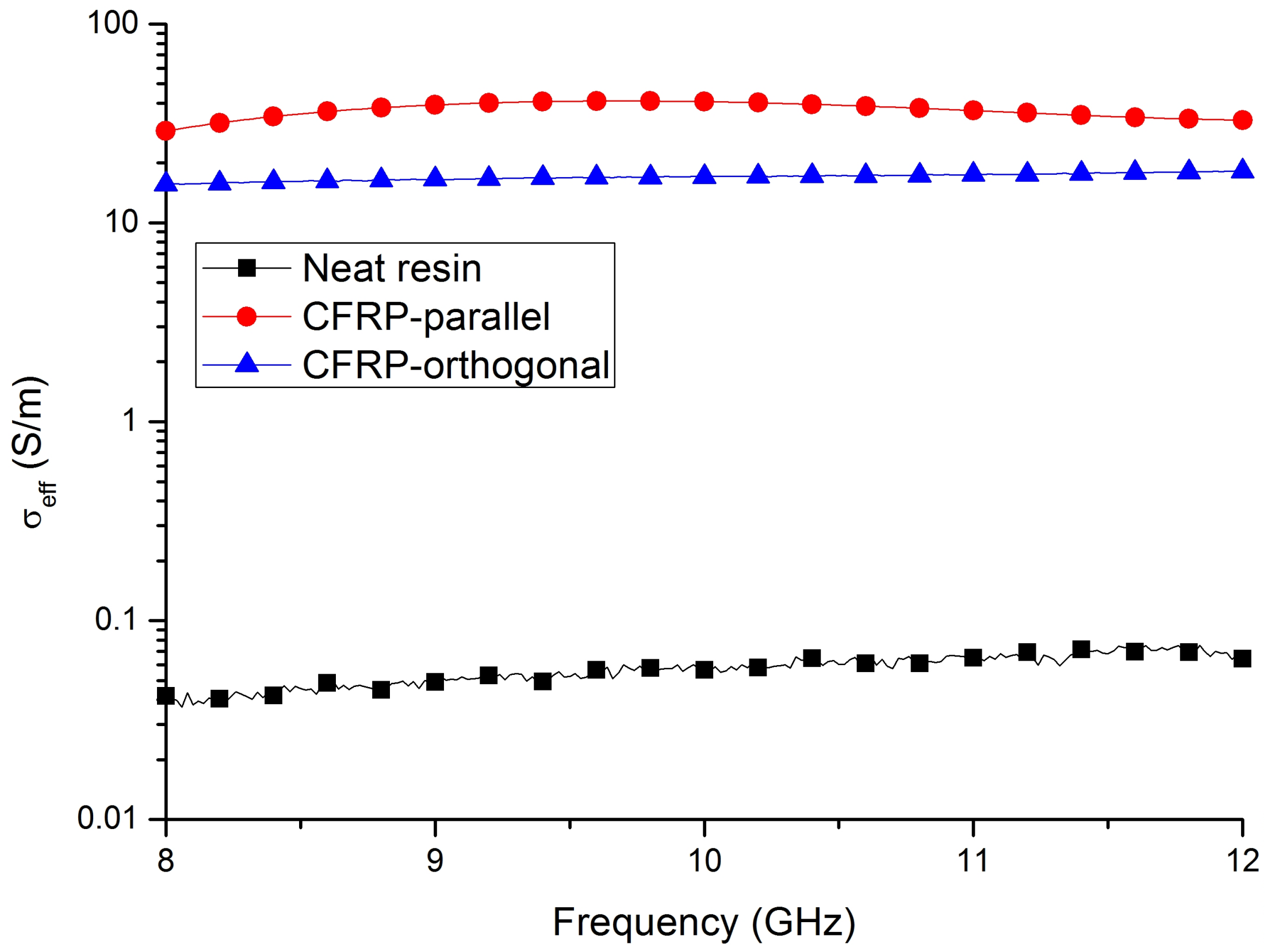




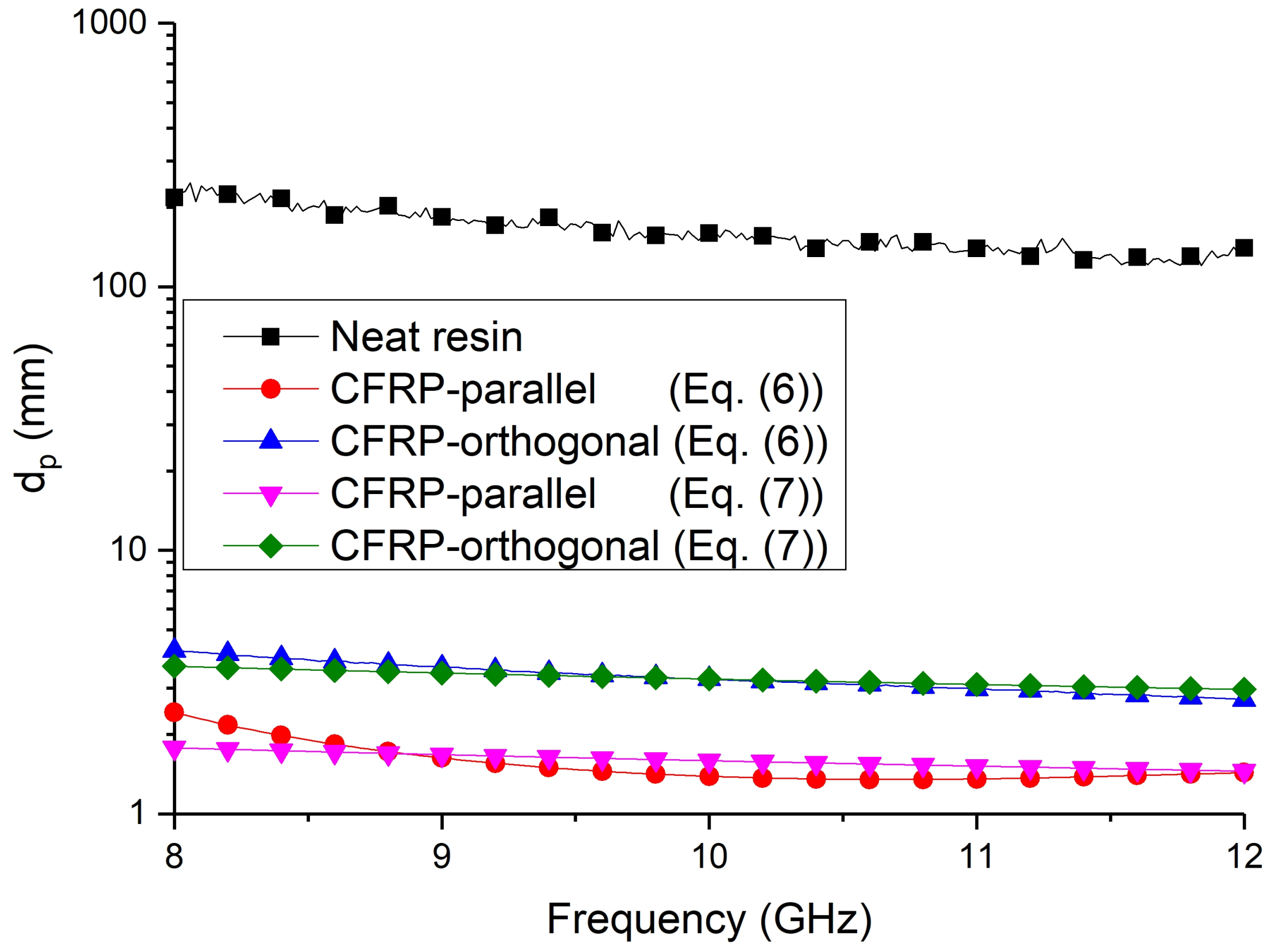




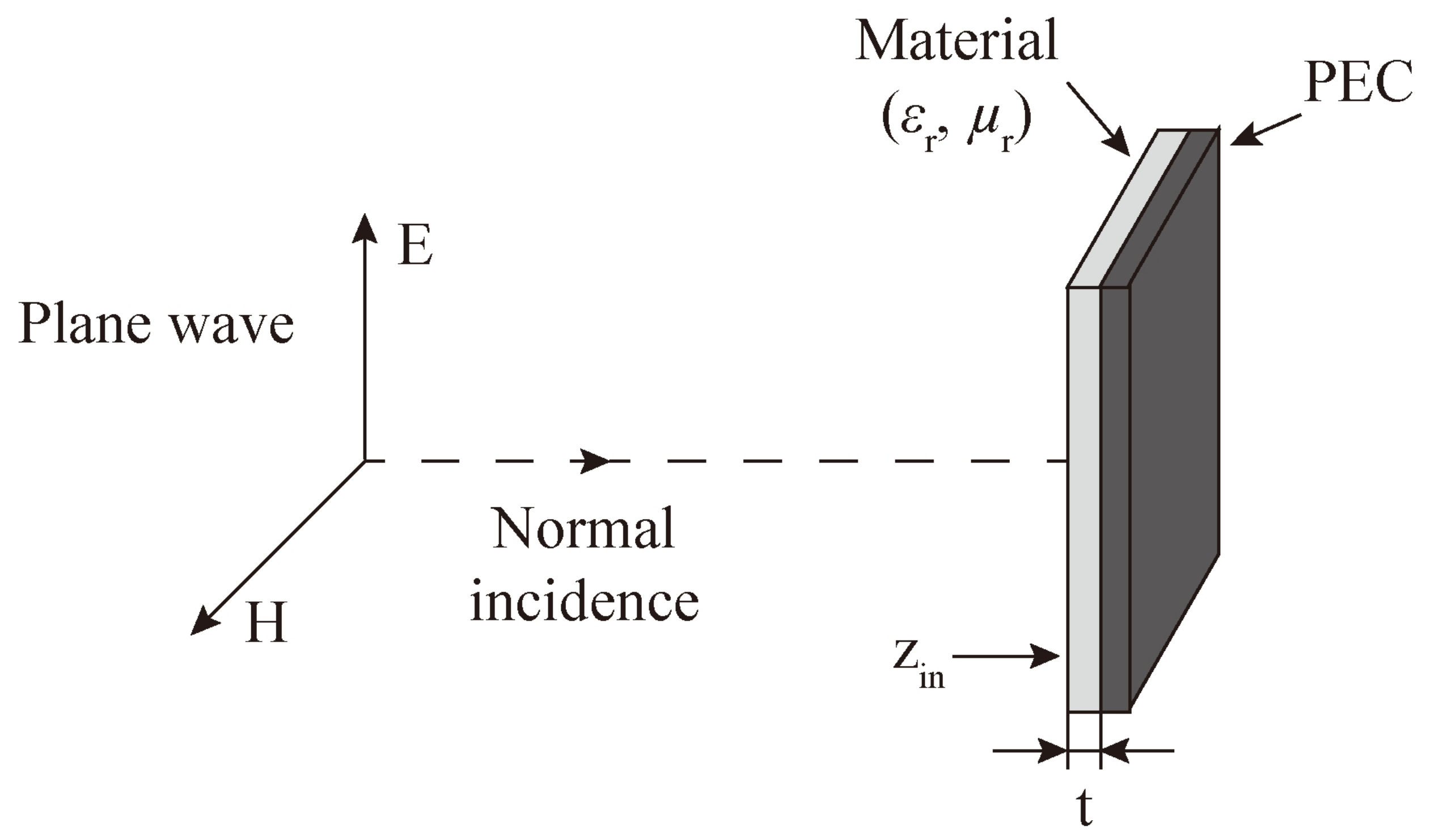




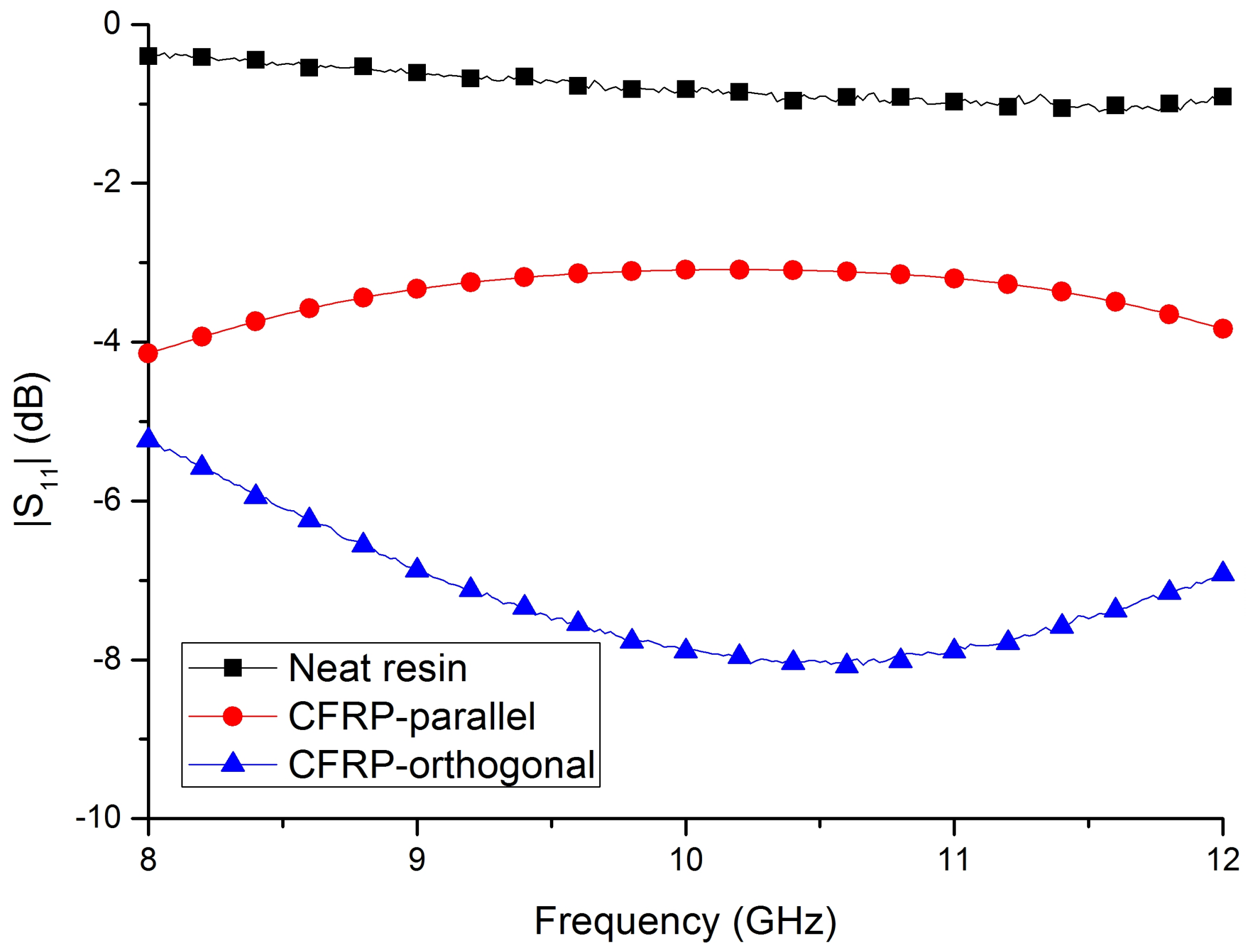

